\title{
Genetic differentiation and estimated levels of gene flow in members of the Gammarus pulex-group (Crustacea, Amphipoda) in western Europe
}

\author{
Maarten Scheepmaker \\ Institute of Taxonomic Zoology, University of Amsterdam, P.O. Box 4766, 1009 AT Amsterdam, \\ The Netherlands
}

Keywords: Taxonomy, Distribution, Population structure, Intra-specific divergence, Gammarus

\begin{abstract}
Genetic differentiation of morphologically variable members of the $G$. pulex-group in northwestern Europe was investigated by electrophoresis at 20 enzyme loci. Five recently described related forms were examined with reference to the "classical" species G. pulex, G. wautieri and G. fossarum. G. pulex and G. wautieri were shown to be genetically homogeneous and clearly distinct species. In the other species, morphologically similar forms were shown to be genetically distant, and inter-areal intraspecific genetic differentiation may warrant recognition of subspecies or sibling species. In some cases, levels of reproductive isolation of the forms involved could be assessed by cross-breeding experiments. To estimate dispersal capabilities, gene flow levels were indirectly estimated from gene frequency data. Gene flow levels are generally low and fluctuating population sizes may enhance the occurrence of stochastic processes. Dendrograms derived from genetic distances were compared with zoogeographical and paleoclimatological evidence. The distribution areas of the species involved correspond to patterns predicted by these data.
\end{abstract}

\section{Résumé}

La différentiation génétique des membres morphologiquement variables du groupe Gammarus pulex dans le nord de l'Europe occidentale a été étudiée par électrophorèse à 20 loci d'enzymes. Cinq formes apparentées récemment décrites ont été étudiée par rapport aux espèces "classiques" $G$. pulex, $G$. wautieri et $G$. fossarum. $G$. pulex et $G$. wautieri se sont montrés des espèces génétiquement homogènes et clairement distinctes. Dans les autres espèces, des formes mor phologiquement similaires se sont montrées génétiquement éloignées, et la différentiation génétique intraspécifique entre aires de répartition différentes peut permettre de reconnaître des sous-espèces ou des espècesjumelles. Dans certains cas, des essais d'hybridation ont permis d'établir des niveaux d'isolation réproductive des formes concernées. Pour une estimation des capacités de dispersion, des niveaux de flux génétique ont été indirectement estimés à partir des données de fréquence des gènes. En général, les niveaux de flux génétique obtenus sont bas et il est probable que l'occurrence des processus stochastiques est favorisée par les fluctuations de l'ordre de grandeur des populations concernées. Des dendrogrammes dérivés des distances génétiques ont été confrontés aux données zoogéographiques et paléoclimatologiques. Les aires de répartition des espèces concernées correspondent bien avec les modèles prédits par ces données.

\section{Introduction}

Due to a considerable amount of morphological variability, the systematic position of the species of the Gammarus pulex-group (sensu Karaman \& Pinkster, 1977) in western Europe has been a source of confusion for quite some time (see Pinkster, 1972). After careful morphological examination and hybridization experiments by several authors (e.g. Wautier \& Roux, 1959; Roux, 1971; Goedmakers, 1972; Pinkster, 1972; Karaman \& Pinkster, 1977) most of the "deviating" forms encountered could be attributed to the "classical" species $G$. pulex (Linnaeus, 1758), G. fossarum Koch, 1835, and G. wautieri A.L. Roux, 1967.

However, three forms were found that are closely related to, but clearly distinguishable from $G$. fossarum (cf. Goedmakers, 1974; Goedmakers \& Roux, 1975; Pinkster, 1983; Pinkster \& Scholl, 1984; Scheepmaker, 1987). These three forms show morphological affinity with G. ibericus Margalef, 1951 , but are reproductively isolated from it (Pinkster, 1983; Pinkster \& Scholl, 1984). Two of these 
Table I. Sampling localities and species distribution.

\begin{tabular}{|c|c|c|c|c|c|c|c|}
\hline $\begin{array}{c}\text { Station } \\
\text { no. }\end{array}$ & $\begin{array}{l}\text { Area in } \\
\text { fig. } 1 \mathrm{~A}\end{array}$ & Species & Country & Prov/dept & $\begin{array}{l}\text { Drainage } \\
\text { system }\end{array}$ & Locality description/ reference & $\begin{array}{r}\text { Sampling } \\
\text { date }\end{array}$ \\
\hline
\end{tabular}

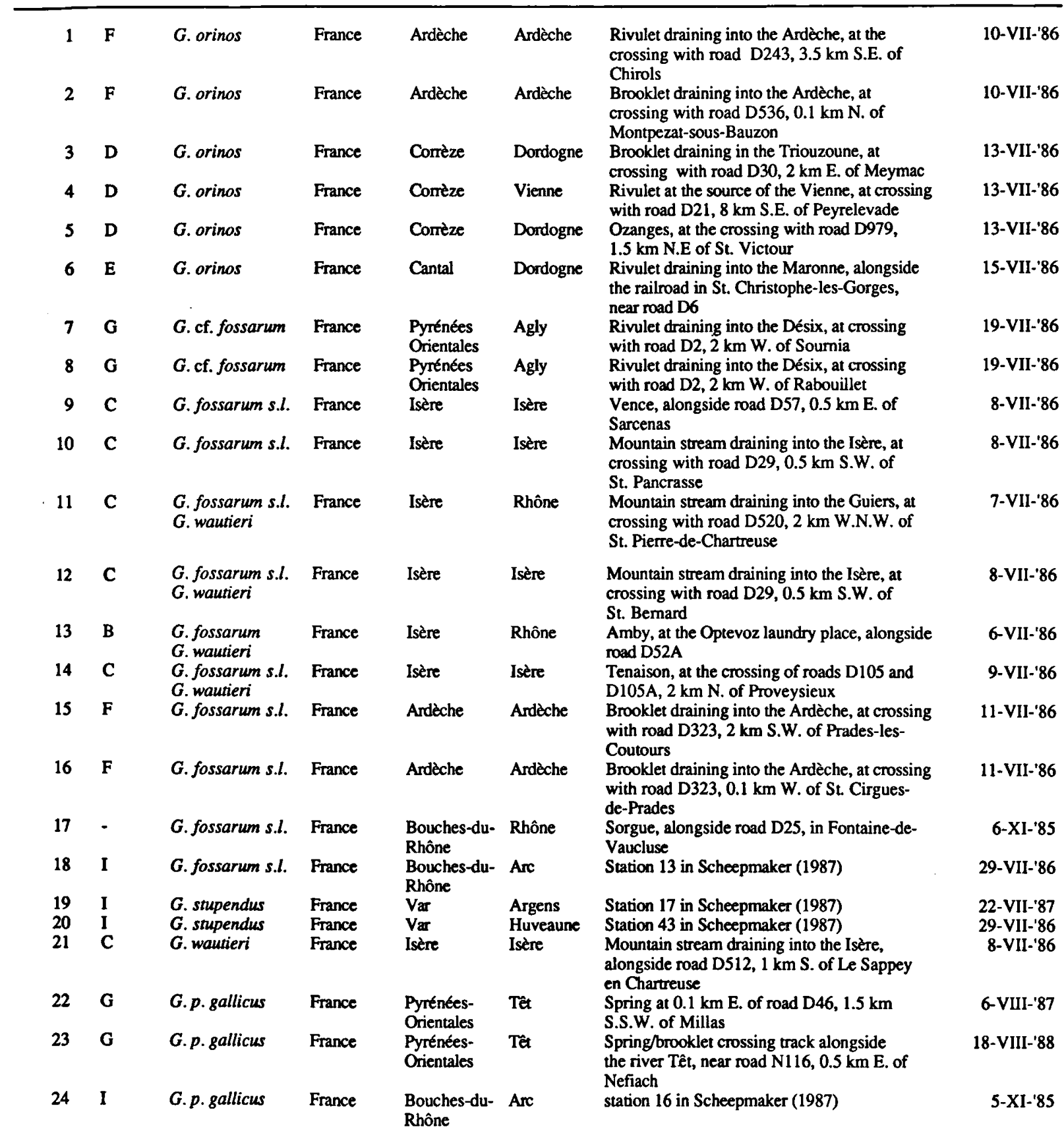


Table l. Cont.

\begin{tabular}{|c|c|c|c|c|c|c|c|}
\hline $\begin{aligned} \text { Station } \\
\text { no. }\end{aligned}$ & $\begin{array}{l}\text { Area in } \\
\text { fig. } 1 \mathrm{~A}\end{array}$ & Species & Country & Prov/dept & $\begin{array}{l}\text { Drainage } \\
\text { system }\end{array}$ & Locality description/ reference & $\begin{array}{r}\text { Sampling } \\
\text { date }\end{array}$ \\
\hline 25 & - & G.p.pulex & France & Loiret & Loire & $\begin{array}{l}\text { Cléry, at crossing with road D32, } 1.5 \mathrm{~km} \\
\text { N.W. of St. Hilaire-les-Andrésis }\end{array}$ & 16-VIII-'88 \\
\hline $\mathbf{a}$ & $\mathbf{F}$ & G. orinos & France & Andèche & Andèche & Station 2 in Scheepmaker et al. (1988) & - \\
\hline b & $\mathbf{H}$ & G. cf. fossarum & France & Ariège & Ariège & Station 3 in Scheepmaker et al. (1988) & - \\
\hline c & $\mathbf{H}$ & G. cf. fossarum & France & Ariège & Ariège & Station 4 in Scheepmaker et al. (1988) & - \\
\hline d & $\mathbf{H}$ & G. cf. fossarum & France & Ariège & Garonne & Station 5 in Scheepmaker et al. (1988) & - \\
\hline e & I & G. stupendus & France & Var & Gapeau & $\begin{array}{l}\text { Station } 1 \text { in Scheepmaker (1987) and } \\
\text { Scheepmaker et al. (1988) }\end{array}$ & - \\
\hline $\mathbf{f}$ & $\mathbf{M}$ & G. fossarum s.str. & Germany & Regensburg & Donau & Station 1 in Scheepmaker \& Van Dalfsen (1989) & - \\
\hline $\mathbf{g}$ & $\mathbf{M}$ & G. fossarum s.str. & Germany & Regensburg & Donau & Station 2 in Scheepmaker \& Van Dalfsen (1989) & - \\
\hline h & $\mathbf{M}$ & G. fossarum s.str. & Germany & Regensburg & Donau & Station 3 in Scheepmaker \& Van Dalfsen (1989) & - \\
\hline $\mathbf{i}$ & $\mathrm{L}$ & G. fossarum s.l. & Switzerland & Oberaargau & Langete & Station 4 in Scheepmaker \& Van Dalfsen (1989) & - \\
\hline $\mathbf{j}$ & L & $\begin{array}{l}\text { G. fossarum s.l. } \\
\text { G. p.pulex }\end{array}$ & Switzerland & Bern & Aare & Station 5 in Scheepmaker \& Van Dalfsen (1989) & - \\
\hline $\mathbf{k}$ & $\mathrm{L}$ & G. fossarum s.l. & Switzerland & Bern & Aare & Station 6 in Scheepmaker \& Van Dalfsen (1989) & - \\
\hline 1 & $\mathbf{K}$ & G. fossarum s.l. & Belgium & Luxembourg & Meuse & Station 7 in Scheepmaker \& Van Dalfsen (1989) & - \\
\hline $\mathbf{m}$ & $\mathbf{K}$ & G. fossarum s.l. & Belgium & Liège & Meuse & Station 8 in Scheepmaker \& Van Dalfsen (1989) & $\cdot$ \\
\hline $\mathbf{n}$ & - & $\begin{array}{l}\text { G. fossarum s.l. } \\
\text { G.p.pulex }\end{array}$ & Belgium & Namur & Escaut & Station 9 in Scheepmaker \& Van Dalfsen (1989) & $\cdot$ \\
\hline $\mathbf{0}$ & - & G. fossarum s.l & Belgium & Brabant & IJzer & Station 10 in Scheepmaker \& Van Dalfsen (1989) & - \\
\hline $\mathbf{p}$ & $\mathbf{J}$ & G. fossarum s.l. & France & Pas-de-Calais & Liane & Station 11 in Scheepmaker \& Van Dalfsen (1989) & - \\
\hline $\mathbf{q}$ & $\mathbf{J}$ & G. fossarum s.l. & France & Pas-de-Calais & Wimereux & Station 12 in Scheepmaker \& Van Dalfsen (1989) & - \\
\hline $\mathbf{r}$ & $\mathbf{J}$ & G. fossarum s.l. & France & Pas-de-Calais & Slack & Station 13 in Scheepmaker \& Van Dalfsen (1989) & - \\
\hline $\mathbf{s}$ & $J$ & $\begin{array}{l}\text { G. fossarum s.l. } \\
\text { G.p.pulex }\end{array}$ & France & Pas -de-Calias & Slack & Station 14 in Scheepmaker \& Van Dalfsen (1989) & - \\
\hline $\mathbf{t}$ & $\cdot$ & G.p.pulex & Netherlands & Gelderland & $\begin{array}{l}\text { Hierdense } \\
\text { beek }\end{array}$ & Station 15 in Scheepmaker \& Van Dalfsen (1989) & $\cdot$ \\
\hline
\end{tabular}

forms were described as $G$. stupendus Pinkster, 1983 and G. orinos Pinkster \& Scholl, 1984. The taxonomic status of the third form (G. cf. fossarum in Goedmakers \& Roux, 1975, and Scheepmaker et al., 1988) has not yet been resolved. All three forms are genetically distinct from one another and from G. ibericus (cf. Pinkster \& Scholl, 1984; Scheepmaker et al., 1988).

Genetic differentiation of $G$. fossarum and $G$. caparti Pètre-Stroobants, 1980 with reference to $G$. pulex was studied by Scheepmaker \& Van Dalfsen (1989). These authors proposed the term G. fossarum sensu lato for all populations hitherto identified as G. fossarum (including G. caparti) and reserved $G$. fossarum sensu stricto for the genetically distinct populations from the area surrounding the former type locality near Regensburg (Federal Republic of Germany).

This study primarily aims at resolving levels of genetic divergence among populations of $G$. fossa- rum sensu lato (cf. Scheepmaker \& Van Dalfsen, 1989), G. orinos, G. stupendus, and G. cf. fossarum, with reference to the "classical" species $G$. pulex - including G. pulex gallicus (S. Karaman, 1931) - and $G$. wautieri. In addition, genetic differentiation was studied in relation to indirectly estimated levels of gene flow. Secondly, the relation between morphological and genetic differentiation among populations of the above species was investigated.

Genetic variation at 20 presumptive gene loci was studied using starch gel enzyme electrophoresis. The evolutionary significance of the genetic variation recorded could be evaluated in part with the results from hybridization experiments by Roux (1971), Goedmakers (1972), Goedmakers \& Roux (1975), Pinkster (1983) and Scheepmaker (1987). In addition to these experiments, crosses have been carried out between four population samples of $G$. fossarum sensu lato (including $G$. caparti), one of 

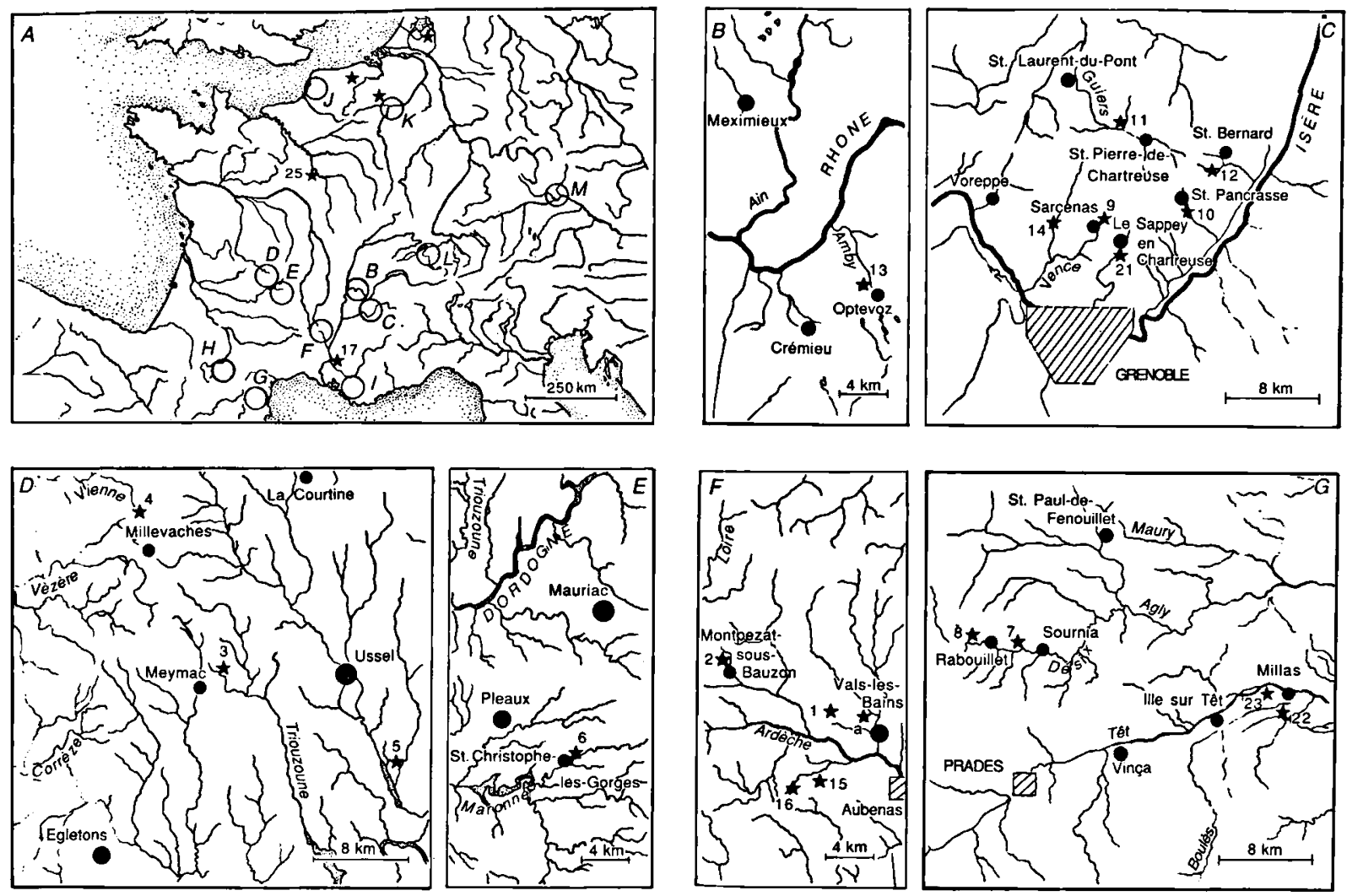

Fig. IA-G. Study areas and sampling localities (cf. Table I).

G. orinos and one of $G$. cf. fossarum.

\section{Material and methods}

\section{Sampling and collection sites}

Sampling for electrophoretic studies and crossbreeding experiments has been carried out according to the procedures of Scheepmaker (1987). In addition to sampling by Scheepmaker et al., 1988 (Fig. 1A, areas F, H and I; Fig. 1F, station a; Table I, stations a-e) and Scheepmaker \& Van Dalfsen, 1989 (Fig. 1A, areas J-M, stations i, j and t; Table I, stations $\mathrm{f}-\mathrm{t}$ ), G. fossarum s.l., G. orinos, G. cf. fossarum, and $G$. $p$. pulex were sampled at 25 further localities. Moreover, G. wautieri and $G . p$. gallicus were sampled at 5 and 3 localities, respectively. The collection sites, sampling dates and the species collected are listed in Table I and Fig. 1. For detailed maps of areas I, $\mathrm{H}$ and F-M in Fig. $1 \mathrm{~A}$ is

referred to Scheepmaker (1987), Scheepmaker et al. (1988), and Scheepmaker \& Van Dalfsen (1989), respectively.

For a discussion and figures of morphological variation of $G$. fossarum s.l. in areas $\mathrm{J}, \mathrm{K}, \mathrm{L}$ and M (Fig. 1A) is referred to Scheepmaker \& Van Dalfsen (1989). G. fossarum s.l. from areas B and C (Fig. 1A) differs from G. fossarum s.l. population samples from other areas primarily by the total absence of calceoli on the A2. The setation on the peduncle segments and flagellum of the A2 is regular, comb-like, often longer than the segments on which they are implanted (for figures see Karaman \& Pinkster, 1977). Individuals from area F resemble those from area L (Fig. 1A); individuals from station 17 are rather similar to those of areas B and $\mathrm{C}$, but most individuals bear calceoli; and individuals from station 18 (Fig. 1, area I) resemble individuals from area $\mathrm{J}$ (Fig. 1A). The morphological variation of some characters (cf. Scheepmaker \& 
Table II. Morphological differentiation of selected variable characters in G. fossarum s.l. Legend: $\gg$ setae much longer than the diameter of the segment on which they are implanted; $>=$ setae longer or equal to the diameter of the segment on which they are implanted; $<=>$ setae shorter, equal or longer than the diameter of the segment on which they are implanted; - setation poor; + setation moderate; ++ setation dense.

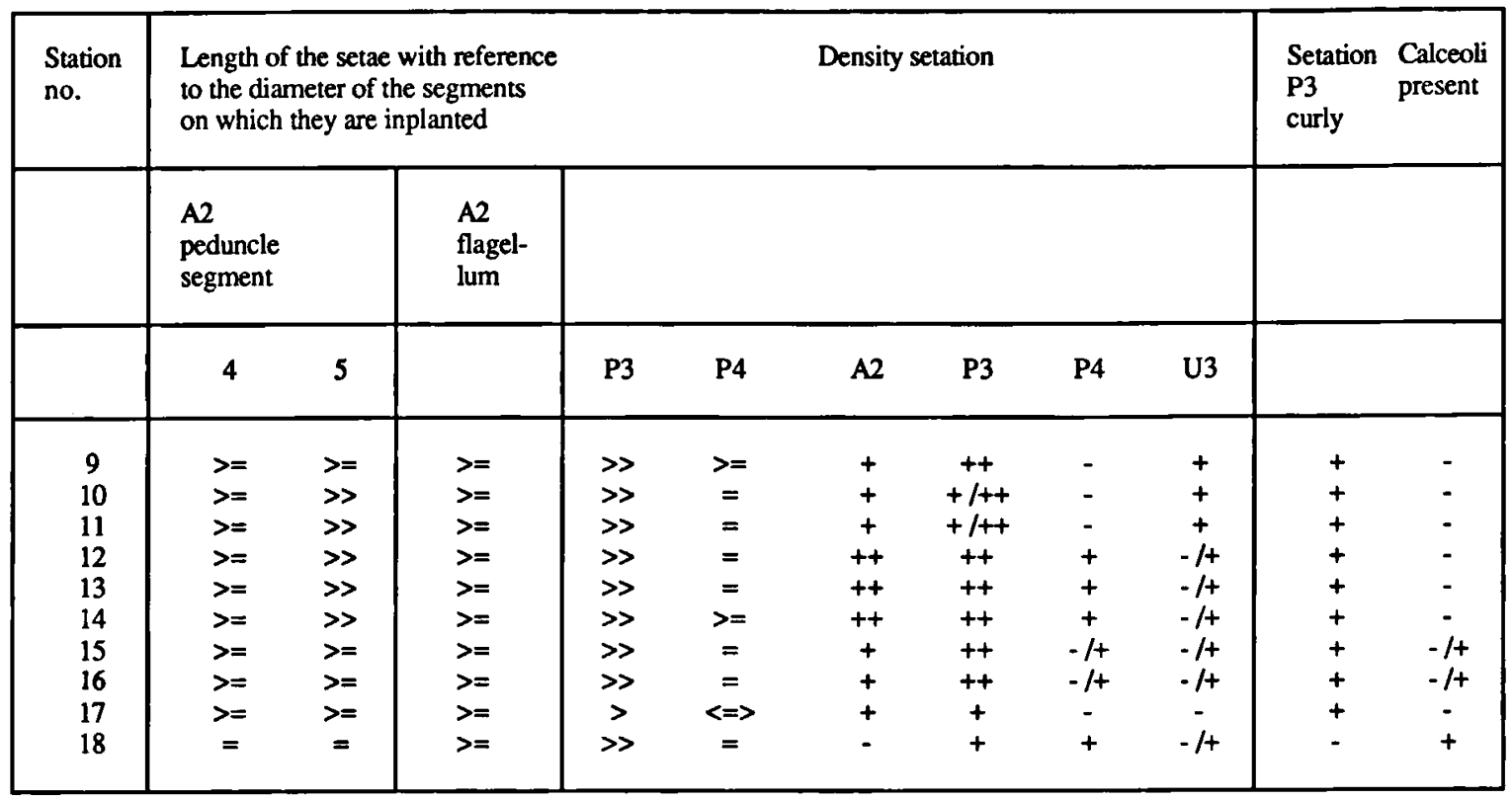

Van Dalfsen, 1989) of G. fossarum s.l. in population samples from areas $B, L, F$, and I (+ station 17) is listed in Table II.

Morphological differentiation of the related forms $G$. orinos, $G$. cf. fossarum and $G$. stupendus of areas F, H, and I was discussed by Pinkster \& Scholl (1984), Scheepmaker et al. (1988), Pinkster (1983) and Scheepmaker (1987). Populations of $G$. cf. fossarum from area $\mathrm{G}$ (Fig. 1A) are morphologically similar to those of area $\mathrm{H}$; likewise, populations of $G$. orinos from areas D and $\mathrm{E}$ are similar to those of area $F$. The most conspicuous difference between $G$. wautieri and $G$. fossarum s.l. is the length of the inner ramus of uropod $3 \mathbf{7 5 \%}$ of the length of the outer ramus for $G$. wautieri, vs. $50 \%$ for $G$. fossarum). In females of $G$. wautieri the setation of the peduncle segments 4 and 5 is shorter than in females of $G$. fossarum (shorter than the diameter of the segment on which they are implanted), and the setae are implanted in a more irregular way. However, the younger females in particular are difficult to distinguish from G. fossarum s.l.

Morphological differentiation of G.p.pulex and G. p. gallicus vs. G. fossarum s.l. and $G$. wautieri was reviewed by Karaman \& Pinkster (1977). The morphological differentiation of some taxonomically important characters among these species and forms is summarized in Table III; for figures see Karaman \& Pinkster (1977) and Scheepmaker et al. (1988).

Stations 18-20 (Fig. 1A, area I) correspond to stations 13, 17 and 43 in Scheepmaker (1987), and therefore they are not figured on a detailed map. As this study involved only 4 enzyme loci, new population samples were taken and analyzed. Stations 15-17 were sampled to evaluate inter-specific, intra-areal comparisons between on the one hand local G. fossarum s.l. and G. orinos from area F, and on the other G. fossarum s.l. and G. stupendus from area $I+$ station 17. Stations 9-14 include the localities where Roux (1971) and Goedmakers (1972) collected individuals of $G$. fossarum and 
G. wautieri for cross-breeding experiments (viz. my stations 9 and 13 respectively).

\section{Cross-breeding experiments}

Cross-breeding experiments followed Pinkster (1983) and were carried out with samples of (a) $G$. fossarum s.l. (G. caparti) from the type locality of G. caparti (area K, station l), G. fossarum s.l. from area $\mathrm{J}$ (station $\mathrm{r}$ ), G. fossarum s.l. from area L (station k), G. orinos from the type locality (area F, station a), and $G$. cf. fossarum from area $\mathrm{H}$ (station b). All combinations of crosses were performed, except for crosses among samples of $G$. orinos and $G$. fossarum s.l. from stations a and $\mathrm{r}$, which were carried out by Pinkster \& Scholl (1984).

\section{Electrophoresis}

Electrophoresis and staining procedures were identical to those of Scheepmaker et al. (1988). The following enzymes (E.C. numbers between parentheses) were assayed: ADA - Adenosine deaminase (3.5.4.4); ALP-Alkaline phosphatase (3.1.3.1); APK - Arginine phosphate kinase (2.7.3.3); EST Esterase (3.1.1.1); GDH-Glutamate dehydrogenase (1.1.1.47); GOT-Glutamic oxaloacetic transaminase (2.6.1.1); GPI - Glucose phosphate isomerase (5.3.1.11); HK - Hexokinase (2.7.1.1); LAP - Leucine aminopeptidase (3.4.1.1); $\mathrm{MDH}$ Malate dehydrogenase (1.1.1.37); ME - Malic enzyme (1.1.1.40); MPI - Mannose phosphate isomerase (5.3.1.8); PEP-Peptidase (3.4.11/13); 6PGD-6 Phosphogluconate dehydrogenase (1.1.1.44); PK - Pyruvate kinase (2.7.1.40).

\section{Analysis of allozyme variation}

The genetic interpretation of the variation was inferential. Data were analyzed by the computer program BIOSYS-1 (Swofford \& Selander, 1981). Matrices of Rogers' (1972) genetic distance, and the genetic distance and -identity according to Nei (1972; $D$ and $I$ respectively) were calculated for all population samples, including the French samples studied by Scheepmaker et al., 1988 (stations a-e) and samples studied by Scheepmaker $\&$ Van Dalfsen, 1989 (stations $\mathrm{f}-\mathrm{t}$ ). From these data, an UPGMA dendrogram (Sneath \& Sokal, 1973) and a distance Wagner network (Farris, 1972) were constructed (for sake of space, complete distance matrices are not presented here).

To measure intra-specific differentiation, $\theta$ values according to Weir \& Cockerham (1984) were calculated with the aid of a Macintosh computer program produced by W.N. Ellis (1989). For comparison, $F_{S T}$-values according to Wright (1931, 1951, 1965) were also calculated. For loci with multiple alleles, a weighted average $\theta$ (and $F_{S T}$ ) was calculated. Significance of the observed $\theta$-values was tested with a chi-square heterogeneity test (Workman \& Niswander, 1970, in Waples, 1987). Quantitative gene flow estimates $N m_{F_{S T}}$ and $N m_{\theta}$ were derived from the relationship $F_{S T}=$ $1 /(1+4 N m)$ in which $N$ is the effective population size and $m$ the migration rate (Wright, 1931). As indices of gene flow based on $F$-statistics may be influenced by natural selection and therefore may constitute poor indicators of the genetic structure of populations, these indices were used in combination with the methods of Slatkin $(1981,1985)$. These methods do not depend on the assumption of selective neutrality (Barton \& Slatkin, 1987). Gene flow levels among populations and areas were also calculated from the average frequency of private alleles, $\vec{p}(1)$, using Slatkin's (1985) formula:

$$
N m=e^{\frac{-\ln \{\lfloor p(1)]+2.44\}}{0.505}}
$$

This formula was derived empirically from simulation experiments where $N=25$. To adjust for differences in sample size, a corrected $\mathrm{Nm}$ value, $\mathrm{Nm}^{*}$, is obtained by dividing the $\mathrm{Nm}$ estimate by the ratio of the actual average sample size to 25 (Slatkin, 1985):

$N m^{*}=\frac{25 N m}{N_{\text {act }}}$, in which $N_{\text {act }}$ is the actual average sample size. 
Table III. Morphological differentiation of some taxonomically important characters in G. orinos, G. cf. fossarum, G. stupendus, G. fossarum s.l., G. wautieri, G. p. pulex and G. p. gallicus.

\begin{tabular}{|c|c|c|c|c|c|c|c|}
\hline species & G. orinos & G. cf. fossarum & G. stupendus & G. fossarum s.l. & G. wautieri & G.p.pulex & G.p. gallicus \\
\hline Setosity A2 & moderate & moderate & moderate & little & little & dense & little \\
\hline Flagellum swollen & - & - & - & - & - & + & + \\
\hline Calceoli & + & - & - & $-1+$ & + & + & + \\
\hline Setosity P5-7 & long & moderate & long / moderate & little & absent & absent & absent \\
\hline Setae longer than spines & ++ & + & $-1+1++$ & - & - & - & - \\
\hline Uropod 3: endop./exop. & $1 / 2$ & $1 / 2$ & $1 / 2$ & $1 / 2$ & $3 / 4$ & $3 / 4$ & $3 / 4$ \\
\hline Urosomites elevated & - & - & - & - & - & - & + \\
\hline
\end{tabular}

Conditional average frequency (CAF; Slatkin, 1981) gene flow profiles were obtained by plotting the average values, $\bar{p}(i)$, of electromorphs having the same incidence $(i / d$, where $i$ is the number of population samples an electromorph appears in, and $d$ the total number of populations sampled), against their incidence or occupancy number (Slatkin, 1981; Caccone, 1985; Caccone \& Sbordoni, 1987). These procedures have been applied to the complete dataset of a species and to different subsets of populations. CAF gene flow profiles and $\bar{p}$ (1)-values have not been calculated for subsets of less than 4 population samples.

\section{Results}

Twenty presumptive gene loci from 17 enzyme systems were scorable in all populations. Electromorph frequencies and relative mobilities of the enzymes coded for by these loci are listed in Table IV. Electromorphs of the Ada, Alp-1, Alp-2, Apk, $G d h, 6 P g d$ and Lap loci were monomorphic and fixed for the same electromorph in all population samples. The number of isozyme loci and quaternary structures resolved were consistent with the interpretation of Scheepmaker et al. (1988).

\section{Genetic variation among populations}

Differences in electromorph distribution among the populations sampled for this study are shown in
Table IV. The Est-1 ${ }^{\mathrm{c}}$ and Got-1f electromorph almost completely discriminated $G$. p. pulex and $G$. p. gallicus from all other samples involved. $G$. $p$. pulex and G. p. gallicus were also characterized by the occurrence of the $M \mathrm{Mi}^{d}$ and in most cases the $P k^{b}$ electromorphs, and the $P e p-2^{c}$ electromorph was only found in $G$. p. pulex.

$G$. wautieri is characterized in most comparisons by the $H k^{c}$ (also characterizing G. fossarum s.l. from area F), $\mathbf{M p i}^{i}$ (shared with G. fossarum s.l. from area F and station 17), $P k^{d}, P e p-2^{m}$ (except for $G$. wautieri from station 14), and Pep- $4^{h}$ electromorphs. $G$. orinos from area $\mathrm{F}$ is characterized by the monomorphic $\mathrm{Mpi}^{h}$ electromorph. The Est $-I^{e}$ electromorph completely discriminates $G$. cf. fossarum from area $\mathrm{G}$ from all other samples (including $G$. cf. fossarum from area $\mathrm{H}$ ); these samples (and $G$. cf. fossarum from area $\mathrm{H}$; Scheepmaker et al., 1988) are also characterized by the $G p i^{k}$ electromorph. The occurrence of the $G p i^{i}$ electromorph is limited to $G$. wautieri (areas B, C) and one sample of G. stupendus (station 19, area I). Local populations may deviate from the general pattern (thus contributing to a discontinuous local variation pattern) by the occurrence of typical and highly frequent electromorphs (e.g. Pep- ${ }^{l}$ and $M d h-I^{e}$ in G. fossarum s.l. from station 18, area I; Table I, Fig. 1A; Got-1' and $G i^{g}$ in G. stupendus from station 19 , area I).

Differences between numbers of loci that are discriminative or diagnostic ( 0.99 criterion; Ayala \& Powell, 1972) among pairwise comparisons of species or forms by area (including data from Scheep- 
Table IV. Electromorph frequencies at 13 presumptive gene loci. $o=G$. orinos; $\mathrm{cf} . f=G$. cf. fossarum; $f=G$. fossarum; $s=G$. stupendus; $w=G$. wautieri; $g=G$. $p$. gallicus; $p=G$. $p$. pulex; $\mathrm{N}=$ sample size; $h=$ heterozygosity per locus (direct count); $H$ $=$ mean heterozygosity over all (including monomorphic) loci; $*$ = significant departure from Hardy-Weinberg distribution $(P<0.05)$; electromorph designation from fastest to slowest in alphabetical order, in accordance with Scheepmaker \& Van Dalfsen (1989); in parentheses: alternative allelic designation employed by Scheepmaker et al., 1988.

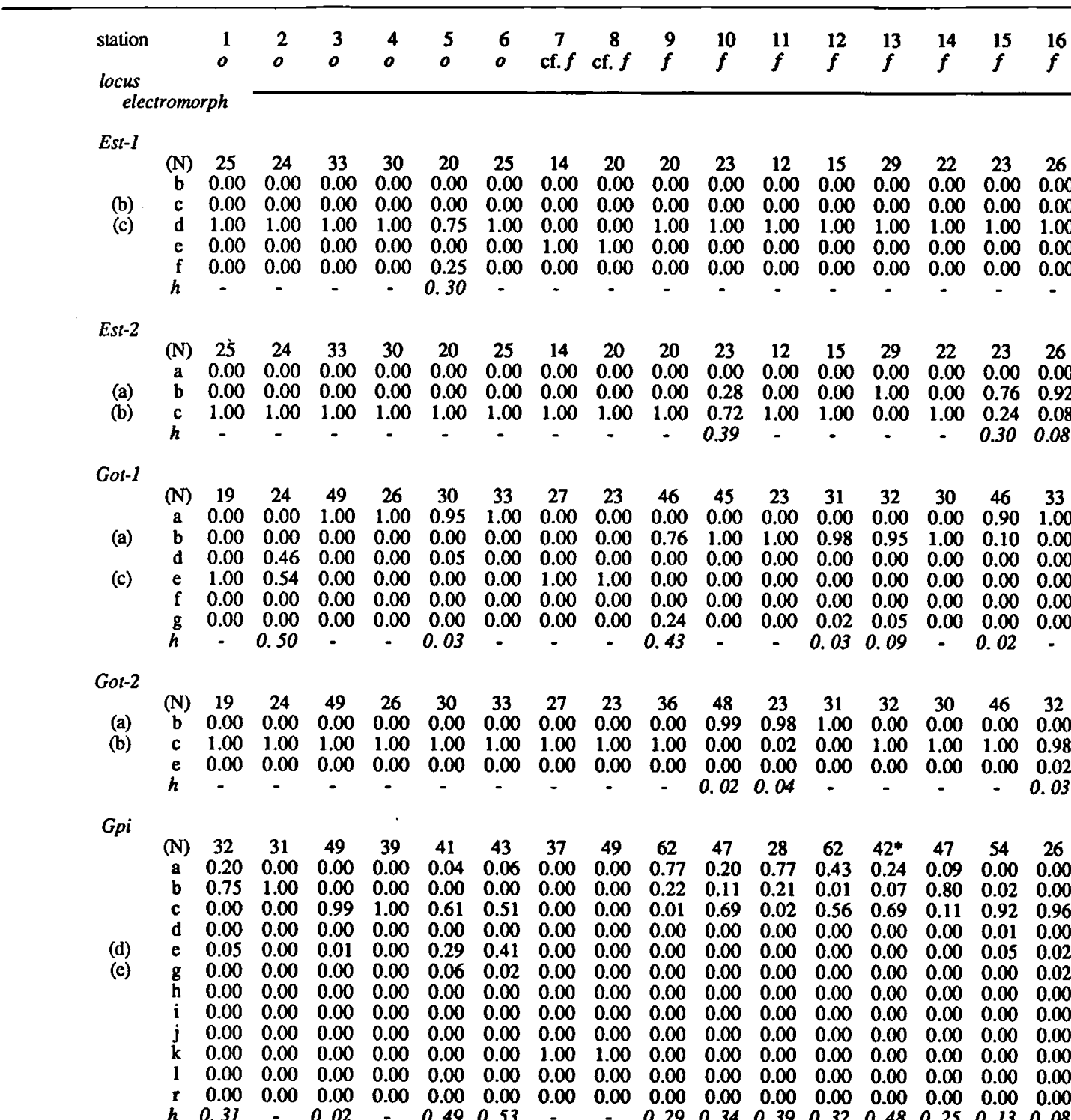

$H \boldsymbol{k}$

(N) $\quad \begin{array}{llllllllllllllll}50 & 38 & 23 & 40 & 40 & 43 & 28 & 33 & 52 & 35 & 16 & 29 & 28 & 11 & 43 & 18\end{array}$

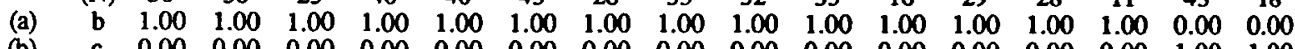

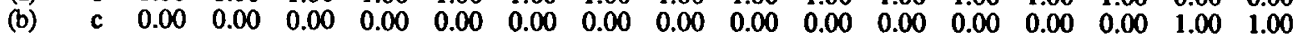

Mdh-1

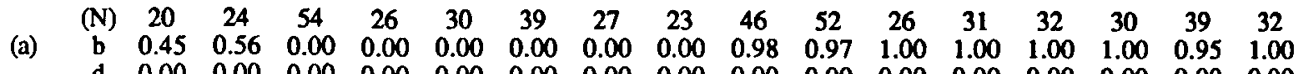

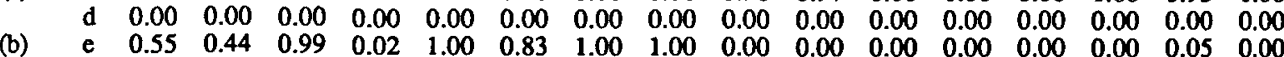
$\begin{array}{llllllllllllllllll}\text { (b) } & \text { e } & 0.55 & 0.44 & 0.99 & 0.02 & 1.00 & 0.83 & 1.00 & 1.00 & 0.00 & 0.00 & 0.00 & 0.00 & 0.00 & 0.00 & 0.05 & 0.00 \\ \text { (c) } & \text { f } & 0.00 & 0.00 & 0.01 & 0.98 & 0.00 & 0.17 & 0.00 & 0.00 & 0.02 & 0.03 & 0.00 & 0.00 & 0.00 & 0.00 & 0.00 & 0.00\end{array}$ $\begin{array}{ccccccccccccccccc}g & 0.00 & 0.00 & 0.00 & 0.00 & 0.00 & 0.00 & 0.00 & 0.00 & 0.00 & 0.00 & 0.00 & 0.00 & 0.00 & 0.00 & 0.00 & 0.00 \\ h & 0.40 & 0.71 & 0.02 & 0.04 & - & 0.23 & - & - & 0.04 & 0.06 & - & - & - & - & 0.02 & -\end{array}$ 
Table IV. Cont.

\begin{tabular}{|c|c|c|c|c|c|c|c|c|c|c|c|c|c|c|c|c|c|}
\hline \multirow{2}{*}{\multicolumn{3}{|c|}{$\begin{array}{lr}\text { station } & 1 \\
\text { locus } & 0 \\
\text { electromorph }\end{array}$}} & $\begin{array}{l}2 \\
0\end{array}$ & $\begin{array}{l}3 \\
0\end{array}$ & $\begin{array}{l}4 \\
0\end{array}$ & $\begin{array}{l}5 \\
0\end{array}$ & $\begin{array}{l}6 \\
0\end{array}$ & $\stackrel{7}{\text { cf. } f}$ & $\stackrel{8}{\text { cf. } f}$ & $\stackrel{9}{f}$ & $\begin{array}{l}10 \\
f\end{array}$ & $\begin{array}{l}11 \\
f\end{array}$ & $\begin{array}{l}12 \\
f\end{array}$ & $\begin{array}{l}13 \\
f\end{array}$ & $\begin{array}{l}14 \\
f\end{array}$ & $\begin{array}{l}15 \\
f\end{array}$ & $\begin{array}{l}16 \\
f\end{array}$ \\
\hline & & & & & & & & & & & & & & & & & \\
\hline \multirow[t]{6}{*}{$M d h-2$} & & & & & & & & & & & & & & & & & \\
\hline & (N) & $\begin{array}{c}19 \\
000\end{array}$ & $\begin{array}{c}24 \\
0.00\end{array}$ & $\begin{array}{c}54 \\
0.00\end{array}$ & $\begin{array}{c}26 \\
0.00\end{array}$ & $\begin{array}{c}30 \\
0.00\end{array}$ & $\begin{array}{c}33 \\
0.00\end{array}$ & $\begin{array}{c}27 \\
0.00\end{array}$ & $\begin{array}{c}23 \\
0.00\end{array}$ & & $\begin{array}{c}45 \\
0.00\end{array}$ & $\begin{array}{c}23 \\
0.00\end{array}$ & $\begin{array}{c}31 \\
0.00\end{array}$ & $\begin{array}{c}32 \\
0.00\end{array}$ & $\begin{array}{c}30 \\
0.00\end{array}$ & $\begin{array}{c}45 \\
0.01\end{array}$ & \\
\hline & b & $\begin{array}{l}0.00 \\
0.00\end{array}$ & $\begin{array}{l}0.00 \\
0.00\end{array}$ & 0.00 & 0.00 & 0.00 & 0.00 & 0.00 & 0.00 & $\begin{array}{l}0.03 \\
0.00\end{array}$ & 0.00 & 0.00 & 0.00 & $\begin{array}{l}0.00 \\
0.00\end{array}$ & $\begin{array}{l}0.00 \\
0.00\end{array}$ & $\begin{array}{l}0.01 \\
0.00\end{array}$ & $\begin{array}{l}0.00 \\
0.00\end{array}$ \\
\hline & c & 0.97 & 1.00 & 1.00 & 1.00 & 1.00 & 1.00 & 1.00 & 1.00 & 0.97 & 1.00 & 1.00 & 1.00 & 1.00 & 1.00 & 0.99 & 1.00 \\
\hline & e & 0.03 & 0.00 & 0.00 & 0.00 & 0.00 & 0.00 & 0.00 & 0.00 & 0.00 & 0.00 & 0.00 & 0.00 & 0.00 & 0.00 & 0.00 & 0.00 \\
\hline & $h$ & 0.05 & - & - & - & - & - & 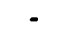 & - & 0.07 & - & - & - & - & - & 0.20 & - \\
\hline \multirow[t]{5}{*}{$M e$} & & & & & & & & & & & & & & & & & \\
\hline & $\underset{\mathrm{b}}{(\mathrm{N})}$ & $\begin{array}{c}28 \\
1.00\end{array}$ & $\begin{array}{c}37 \\
1.00\end{array}$ & $\begin{array}{c}47 \\
1.00\end{array}$ & $\begin{array}{c}61 \\
0.00\end{array}$ & $\begin{array}{c}43 \\
1.00\end{array}$ & $\begin{array}{c}41 \\
1.00\end{array}$ & $\begin{array}{c}36 \\
0.00\end{array}$ & $\begin{array}{c}39 \\
0.00\end{array}$ & $\begin{array}{c}50 \\
0.00\end{array}$ & $\begin{array}{c}56 \\
0.00\end{array}$ & $\begin{array}{c}25 \\
0.00\end{array}$ & $\begin{array}{c}14 \\
0.00\end{array}$ & $\begin{array}{c}43 \\
0.00\end{array}$ & $\begin{array}{c}35 \\
0.00\end{array}$ & $\begin{array}{c}33 \\
0.00\end{array}$ & $\begin{array}{c}30 \\
0.00\end{array}$ \\
\hline & c & 0.00 & 0.00 & 0.00 & 1.00 & 0.00 & 0.00 & 1.00 & 1.00 & 1.00 & 1.00 & 1.00 & 1.00 & 1.00 & 1.00 & 1.00 & 1.00 \\
\hline & d & 0.00 & 0.00 & 0.00 & 0.00 & 0.00 & 0.00 & 0.00 & 0.00 & 0.00 & 0.00 & 0.00 & 0.00 & 0.00 & 0.00 & 0.00 & 0.00 \\
\hline & e & 0.00 & 0.00 & 0.00 & 0.00 & 0.00 & 0.00 & 0.00 & 0.00 & 0.00 & 0.00 & 0.00 & 0.00 & 0.00 & 0.00 & 0.00 & 0.00 \\
\hline \multirow[t]{5}{*}{$M p i$} & & & & & & & & & & & & & & & & & \\
\hline & $(\mathrm{N})$ & 27 & 31 & 52 & 39 & 44 & 35 & 37 & 40 & 62 & 55 & 23 & $45^{*}$ & 41 & 46 & 62 & 26 \\
\hline & b & 0.00 & 0.00 & 0.00 & 0.00 & 0.00 & 0 & 0 & 0.00 & 0.00 & 0.00 & 0 & 0.00 & 0.00 & 0.00 & 0 & 0.00 \\
\hline & d & 0.00 & 0.00 & 0.00 & 0.00 & 0.00 & 0.00 & 0.00 & 0.00 & 0.00 & 0.00 & 0.00 & 0.00 & 0.00 & 0.00 & 0.00 & 0.00 \\
\hline & e & 0.00 & 0.00 & 0.00 & 0.00 & 0.00 & 0.00 & 0.00 & 0.00 & 0.00 & 0.00 & 0.00 & 0.00 & 0.00 & 0.00 & 0.00 & 0.00 \\
\hline \multirow[t]{4}{*}{ (b) } & f & 0.00 & 0.00 & 0.00 & 0.00 & 0.00 & 0.00 & 0.00 & 0.00 & 0.00 & 0.00 & 0.00 & 0.00 & 0.00 & 0.00 & 0.00 & 0.00 \\
\hline & h & 1.00 & 1.00 & 0.02 & 0.00 & 0.00 & 0.00 & 0.00 & 0.00 & 0.00 & 0.00 & 0.00 & 0.00 & 0.00 & 0.00 & 0.00 & 0.00 \\
\hline & i & 0.00 & 0.00 & 0.00 & 0.00 & 0.49 & 0.39 & 0.00 & 0.00 & 0.00 & 0.06 & 0.00 & 0.00 & 0.00 & 0.00 & 0.97 & 1.00 \\
\hline & $\therefore$ & 0.00 & 0.00 & 0.00 & 0.00 & 0.00 & 0.00 & 0.00 & 0.00 & 0.00 & 0.00 & 0.00 & 0.00 & 0.00 & 0.02 & 0.00 & 0.00 \\
\hline & $\mathbf{k}$ & 0.00 & 0.00 & 0.52 & 1.00 & 0.00 & 0.00 & 0.00 & 0.04 & 0.04 & 0.05 & 0.00 & 0.04 & 0.05 & 0.03 & 0.03 & 0.00 \\
\hline \multirow[t]{4}{*}{ (g) } & $\mathrm{m}$ & 0.00 & 0.00 & 0.46 & 0.00 & 0.51 & 0.60 & 1.00 & 0.95 & 0.94 & 0.30 & 0.24 & 0.34 & 0.93 & 0.94 & 0.00 & 0.00 \\
\hline & $\mathbf{n}$ & 0.00 & 0.00 & 0.00 & 0.00 & 0.00 & 0.01 & 0.00 & 0.01 & 0.00 & 0.59 & 0.00 & 0. & 0.00 & 0.00 & 0.00 & 0.00 \\
\hline & 0 & 0.00 & 0.00 & 0.00 & 0.00 & 0.00 & 0.00 & 0.00 & 0.00 & 0.02 & 0.00 & 0.76 & 0.62 & 0.02 & 0.01 & 0.00 & 0.00 \\
\hline & $n$ & 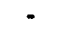 & - & 0.44 & 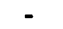 & 0.52 & 0.51 & & 0 & 13 & 0.51 & 0.22 & 0.27 & 0.15 & 0.13 & 0.06 & 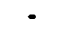 \\
\hline
\end{tabular}

Pep-2

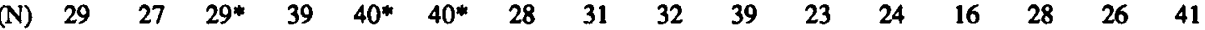
$\begin{array}{ccccccccccccccccc}\text { c } & 0.00 & 0.00 & 0.00 & 0.00 & 0.00 & 0.00 & 0.00 & 0.00 & 0.00 & 0.00 & 0.00 & 0.00 & 0.00 & 0.00 & 0.00 & 0.00\end{array}$ $\begin{array}{lllllllllllllllll}\text { d } & 0.00 & 0.00 & 0.00 & 0.00 & 0.18 & 0.00 & 0.00 & 0.00 & 0.00 & 0.00 & 0.00 & 0.00 & 0.00 & 0.00 & 0.00 & 0.00\end{array}$

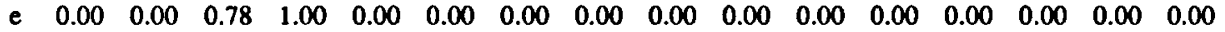
$\begin{array}{lllllllllllllllll}\text { f } & 0.00 & 0.00 & 0.00 & 0.00 & 0.12 & 0.26 & 0.00 & 0.00 & 0.00 & 0.00 & 0.00 & 0.00 & 0.00 & 0.00 & 0.00 & 0.00\end{array}$ $\begin{array}{lllllllllllllllll}\text { h } & 1.00 & 0.00 & 0.00 & 0.00 & 0.70 & 0.74 & 1.00 & 1.00 & 0.00 & 0.00 & 0.00 & 0.00 & 0.00 & 0.00 & 0.00 & 0.00\end{array}$ $\begin{array}{lllllllllllllllll}\text { j } & 0.00 & 1.00 & 0.22 & 0.00 & 0.00 & 0.00 & 0.00 & 0.00 & 0.80 & 1.00 & 0.28 & 0.94 & 1.00 & 0.98 & 1.00 & 1.00\end{array}$ $\begin{array}{lllllllllllllllll}\mathrm{k} & 0.00 & 0.00 & 0.00 & 0.00 & 0.00 & 0.00 & 0.00 & 0.00 & 0.20 & 0.00 & 0.72 & 0.06 & 0.00 & 0.02 & 0.00 & 0.00\end{array}$ $\begin{array}{lllllllllllllllll}1 & 0.00 & 0.00 & 0.00 & 0.00 & 0.00 & 0.00 & 0.00 & 0.00 & 0.00 & 0.00 & 0.00 & 0.00 & 0.00 & 0.00 & 0.00 & 0.00\end{array}$ $\begin{array}{lllllllllllllllll}\mathrm{m} & 0.00 & 0.00 & 0.00 & 0.00 & 0.00 & 0.00 & 0.00 & 0.00 & 0.00 & 0.00 & 0.00 & 0.00 & 0.00 & 0.00 & 0.00 & 0.00\end{array}$ $\begin{array}{lllllllllllllllll}n & 0.00 & 0.00 & 0.00 & 0.00 & 0.00 & 0.00 & 0.00 & 0.00 & 0.00 & 0.00 & 0.00 & 0.00 & 0.00 & 0.00 & 0.00 & 0.00\end{array}$

Pep-4

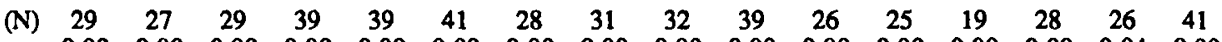

(a) $\quad \begin{array}{ccccccccccccccccc}c & 0.00 & 0.00 & 0.00 & 0.00 & 0.00 & 0.00 & 0.00 & 0.00 & 0.00 & 0.00 & 0.00 & 0.00 & 0.00 & 0.00 & 0.04 & 0.00\end{array}$ $\begin{array}{lllllllllllllllll}\text { d } & 1.00 & 1.00 & 1.00 & 1.00 & 0.89 & 0.92 & 1.00 & 0.95 & 1.00 & 0.90 & 1.00 & 0.58 & 0.92 & 1.00 & 0.96 & 0.92\end{array}$ $\begin{array}{lllllllllllllllll}\text { e } & 0.00 & 0.00 & 0.00 & 0.00 & 0.11 & 0.08 & 0.00 & 0.05 & 0.00 & 0.00 & 0.00 & 0.00 & 0.00 & 0.00 & 0.00 & 0.08\end{array}$ $\begin{array}{lllllllllllllllll}\text { f } & 0.00 & 0.00 & 0.00 & 0.00 & 0.00 & 0.00 & 0.00 & 0.00 & 0.00 & 0.00 & 0.00 & 0.00 & 0.00 & 0.00 & 0.00 & 0.00\end{array}$ $\begin{array}{lllllllllllllllll}\text { g } & 0.00 & 0.00 & 0.00 & 0.00 & 0.00 & 0.00 & 0.00 & 0.00 & 0.00 & 0.00 & 0.00 & 0.00 & 0.03 & 0.00 & 0.00 & 0.00\end{array}$ $\begin{array}{lllllllllllllllll}\mathrm{g} & 0.00 & 0.00 & 0.00 & 0.00 & 0.00 & 0.00 & 0.00 & 0.00 & 0.00 & 0.10 & 0.00 & 0.42 & 0.05 & 0.00 & 0.00 & 0.00\end{array}$

$P k$

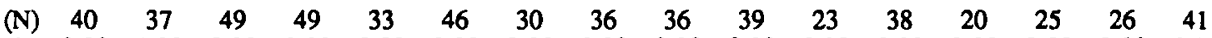
$\begin{array}{ccccccccccccccccc}\text { b } & 0.00 & 1.00 & 0.00 & 0.00 & 0.00 & 0.00 & 0.00 & 0.00 & 0.00 & 0.00 & 0.00 & 0.00 & 0.00 & 0.00 & 0.00 & 0.00\end{array}$

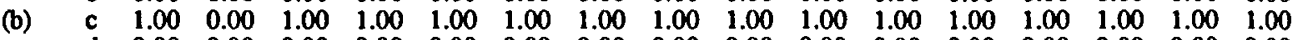
$\begin{array}{lllllllllllllllll}\text { d } & 0.00 & 0.00 & 0.00 & 0.00 & 0.00 & 0.00 & 0.00 & 0.00 & 0.00 & 0.00 & 0.00 & 0.00 & 0.00 & 0.00 & 0.00 & 0.00\end{array}$ $\begin{array}{lllllllllllllllll}\text { e } & 0.00 & 0.00 & 0.00 & 0.00 & 0.00 & 0.00 & 0.00 & 0.00 & 0.00 & 0.00 & 0.00 & 0.00 & 0.00 & 0.00 & 0.00 & 0.00\end{array}$

$\begin{array}{llllllllllllllllllll}H & 0.04 & 0.06 & 0.03 & 0.00 & 0.09 & 0.08 & 0.00 & 0.01 & 0.06 & 0.08 & 0.04 & 0.06 & 0.04 & 0.02 & 0.04 & 0.01\end{array}$ 
Table IV. Cont.

\begin{tabular}{|c|c|c|c|c|c|c|c|c|c|c|c|c|c|c|}
\hline \multirow{2}{*}{\multicolumn{3}{|c|}{$\begin{array}{lc}\text { station } & 17 \\
& f \\
\text { locus } & \\
\text { electromorph }\end{array}$}} & $\begin{array}{l}18 \\
f\end{array}$ & $\begin{array}{l}19 \\
s\end{array}$ & $\begin{array}{l}20 \\
s\end{array}$ & $\begin{array}{l}11 \\
w\end{array}$ & $\begin{array}{l}12 \\
w\end{array}$ & $\begin{array}{l}13 \\
w\end{array}$ & $\begin{array}{l}14 \\
w\end{array}$ & $\begin{array}{l}21 \\
w\end{array}$ & $\begin{array}{l}22 \\
8\end{array}$ & $\begin{array}{l}23 \\
8\end{array}$ & $\begin{array}{l}24 \\
8\end{array}$ & $\begin{array}{l}25 \\
p\end{array}$ \\
\hline & & & & & & & & & & & & & & \\
\hline \multirow[t]{3}{*}{ Est-I } & & & & & & & & & & & & & & \\
\hline & $\begin{array}{c}(\mathrm{N}) \\
\mathrm{b} \\
\mathrm{c} \\
\mathrm{d} \\
\mathrm{e} \\
\mathrm{f}\end{array}$ & $\begin{array}{c}29 \\
0.00 \\
0.00 \\
1.00 \\
0.00 \\
0.00\end{array}$ & $\begin{array}{c}31 \\
0.00 \\
0.00 \\
1.00 \\
0.00 \\
0.00\end{array}$ & $\begin{array}{c}30 \\
0.00 \\
0.00 \\
1.00 \\
0.00 \\
0.00\end{array}$ & $\begin{array}{c}24 \\
0.00 \\
0.00 \\
1.00 \\
0.00 \\
0.00\end{array}$ & $\begin{array}{c}11 \\
0.00 \\
0.00 \\
1.00 \\
0.00 \\
0.00\end{array}$ & $\begin{array}{c}5 \\
0.00 \\
0.00 \\
1.00 \\
0.00 \\
0.00\end{array}$ & $\begin{array}{c}31 \\
0.00 \\
0.00 \\
1.00 \\
0.00 \\
0.00\end{array}$ & $\begin{array}{c}20 \\
0.00 \\
0.00 \\
1.00 \\
0.00 \\
0.00\end{array}$ & $\begin{array}{c}18 \\
0.00 \\
0.00 \\
1.00 \\
0.00 \\
0.00\end{array}$ & $\begin{array}{c}28 \\
0.00 \\
1.00 \\
0.00 \\
0.00 \\
0.00\end{array}$ & $\begin{array}{c}35 \\
0.00 \\
1.00 \\
0.00 \\
0.00 \\
0.00\end{array}$ & $\begin{array}{c}25 \\
0.00 \\
1.00 \\
0.00 \\
0.00 \\
0.00\end{array}$ & $\begin{array}{c}10 \\
0.00 \\
1.00 \\
0.00 \\
0.00 \\
0.00\end{array}$ \\
\hline & $h$ & - & - & - & - & - & - & - & - & - & - & - & - & - \\
\hline
\end{tabular}

Est-2

$\begin{array}{ccccccccccccccc} & \text { (N) } & 29 & 31 & 30 & 24 & 11 & 5 & 31 & 20 & 18 & 28 & 35 & 25 & 10 \\ & \mathrm{a} & 0.00 & 0.00 & 0.00 & 0.00 & 0.00 & 0.00 & 0.00 & 0.00 & 0.00 & 0.00 & 0.00 & 0.00 & 0.00 \\ \text { (a) } & \mathrm{b} & 0.00 & 1.00 & 0.02 & 0.00 & 0.00 & 0.00 & 0.00 & 0.00 & 0.00 & 0.00 & 0.00 & 0.00 & 0.00 \\ \text { (b) } & \mathrm{c} & 1.00 & 0.00 & 0.98 & 1.00 & 1.00 & 1.00 & 1.00 & 1.00 & 1.00 & 1.00 & 1.00 & 1.00 & 1.00 \\ & h & - & - & 0.03 & - & - & - & - & - & - & - & - & - & -\end{array}$

Got-1

$\begin{array}{cccccccccccccc}\text { (N) } & 18 & 12 & 20 & 7 & 3 & 9 & 27 & 9 & 26 & 27 & 25 & 28 & 18 \\ \text { a } & 0.00 & 0.00 & 0.00 & 0.00 & 1.00 & 100 & 0.98 & 1.00 & 1.00 & 0.00 & 0.00 & 0.00 & 0.00\end{array}$

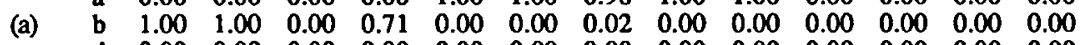

$\begin{array}{llllllllllllll}\text { d } & 0.00 & 0.00 & 0.00 & 0.00 & 0.00 & 0.00 & 0.00 & 0.00 & 0.00 & 0.00 & 0.00 & 0.00 & 0.00\end{array}$

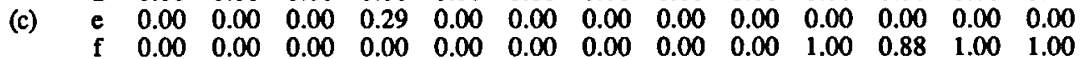

$\begin{array}{llllllllllllll}\mathrm{g} & 0.00 & 0.00 & 1.00 & 0.00 & 0.00 & 0.00 & 0.00 & 0.00 & 0.00 & 0.00 & 0.12 & 0.00 & 0.00\end{array}$

Got-2

$\begin{array}{ccccccccccccccc} & \text { (N) } & 18 & 12 & 20 & 9 & 3 & 9 & 27 & 9 & 26 & 28 & 25 & 28 & 18 \\ \text { (a) } & \text { b } & 0.00 & 0.00 & 0.00 & 0.00 & 0.00 & 0.00 & 0.00 & 0.00 & 0.00 & 0.00 & 0.00 & 0.00 & 0.00 \\ \text { (b) } & \text { c } & 1.00 & 1.00 & 1.00 & 1.00 & 1.00 & 1.00 & 1.00 & 1.00 & 1.00 & 1.00 & 1.00 & 1.00 & 1.00 \\ & \text { e } & 0.00 & 0.00 & 0.00 & 0.00 & 0.00 & 0.00 & 0.00 & 0.00 & 0.00 & 0.00 & 0.00 & 0.00 & 0.00 \\ & h & - & - & - & - & - & - & - & - & - & - & - & - & - \\ \text { Gpi } & & & & & & & & & & & & & & \\ & \text { (N) } & 33 & 30 & 73 & 39 & 20 & 15 & 31 & 30 & 59 & 43 & 44 & 34 & 18 \\ & \text { a } & 0.89 & 0.00 & 0.00 & 0.00 & 0.00 & 0.00 & 0.00 & 0.00 & 0.00 & 0.00 & 0.00 & 0.00 & 0.00 \\ & \text { b } & 0.02 & 1.00 & 0.00 & 0.00 & 0.00 & 0.00 & 0.00 & 0.00 & 0.00 & 0.00 & 0.01 & 0.00 & 0.00 \\ & \text { c } & 0.09 & 0.00 & 0.00 & 0.47 & 0.00 & 0.00 & 0.00 & 0.00 & 0.00 & 0.32 & 0.42 & 0.82 & 0.89 \\ & \text { d } & 0.00 & 0.00 & 0.00 & 0.00 & 0.00 & 0.00 & 0.00 & 0.00 & 0.00 & 0.00 & 0.00 & 0.00 & 0.00 \\ \text { (d) } & \text { e } & 0.00 & 0.00 & 0.02 & 0.44 & 0.00 & 0.00 & 0.00 & 0.00 & 0.00 & 0.04 & 0.36 & 0.07 & 0.00 \\ \text { (e) } & \text { g } & 0.00 & 0.00 & 0.96 & 0.06 & 0.65 & 0.00 & 1.00 & 0.95 & 0.97 & 0.64 & 0.21 & 0.00 & 0.06 \\ & \text { h } & 0.00 & 0.00 & 0.00 & 0.00 & 0.00 & 0.00 & 0.00 & 0.00 & 0.00 & 0.00 & 0.00 & 0.10 & 0.00 \\ & \text { i } & 0.00 & 0.00 & 0.00 & 0.03 & 0.35 & 1.00 & 0.00 & 0.05 & 0.03 & 0.00 & 0.00 & 0.00 & 0.00 \\ & \text { j } & 0.00 & 0.00 & 0.01 & 0.00 & 0.00 & 0.00 & 0.00 & 0.00 & 0.00 & 0.00 & 0.00 & 0.00 & 0.00 \\ & \mathbf{k} & 0.00 & 0.00 & 0.00 & 0.00 & 0.00 & 0.00 & 0.00 & 0.00 & 0.00 & 0.00 & 0.00 & 0.00 & 0.00 \\ & 1 & 0.00 & 0.00 & 0.01 & 0.00 & 0.00 & 0.00 & 0.00 & 0.00 & 0.00 & 0.00 & 0.00 & 0.00 & 0.00 \\ & \text { r } & 0.00 & 0.00 & 0.00 & 0.00 & 0.00 & 0.00 & 0.00 & 0.00 & 0.00 & 0.00 & 0.00 & 0.00 & 0.05 \\ & h & 0.15 & - & 0.10 & 0.38 & 0.20 & - & - & 0.10 & 0.07 & 0.42 & 0.57 & 0.24 & 0.22\end{array}$

$H k$

$\begin{array}{llllllllllllll}\text { (N) } & 39 & 21 & 34 & 39 & 15 & 2 & 25 & 18 & 41 & 33 & 20 & 23 & 13\end{array}$

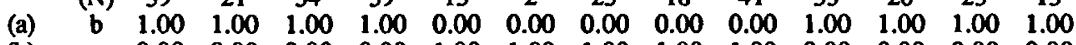

(b) $\quad \begin{array}{llllllllllllll}\text { c } & 0.00 & 0.00 & 0.00 & 0.00 & 1.00 & 1.00 & 1.00 & 1.00 & 1.00 & 0.00 & 0.00 & 0.00 & 0.00\end{array}$

Mdh-1
$\begin{array}{cccccccccccccc}\text { (N) } & 18 & 11 & 20 & 9 & 3 & 9 & 28 & 9 & 26 & 28 & 25 & 38 & 16 \\ \text { b } & 1.00 & 0.00 & 1.00 & 1.00 & 0.00 & 1.00 & 0.91 & 1.00 & 1.00 & 0.93 & 0.84 & 0.97 & 0.53\end{array}$

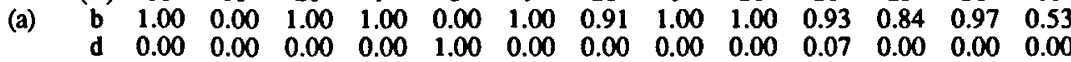
(b) $\quad \begin{array}{lllllllllllllll} & \text { e } & 0.00 & 1.00 & 0.00 & 0.00 & 0.00 & 0.00 & 0.09 & 0.00 & 0.00 & 0.00 & 0.16 & 0.03 & 0.47\end{array}$
(c) $\quad \begin{array}{lllllllllllllll} & \text { f } & 0.00 & 0.00 & 0.00 & 0.00 & 0.00 & 0.00 & 0.00 & 0.00 & 0.00 & 0.00 & 0.00 & 0.00 & 0.00\end{array}$

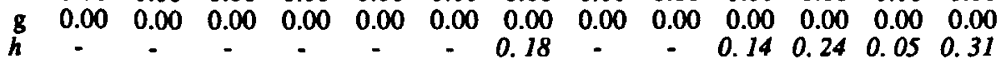


Table IV. cont.

\begin{tabular}{|c|c|c|c|c|c|c|c|c|c|c|c|c|c|c|}
\hline \multicolumn{2}{|l|}{ station } & $\begin{array}{l}17 \\
f\end{array}$ & $\begin{array}{l}18 \\
f\end{array}$ & $\begin{array}{l}19 \\
s\end{array}$ & $\begin{array}{l}20 \\
s\end{array}$ & $\begin{array}{l}11 \\
w\end{array}$ & $\begin{array}{l}12 \\
w\end{array}$ & $\begin{array}{l}13 \\
w\end{array}$ & $\begin{array}{l}14 \\
w\end{array}$ & $\begin{array}{l}21 \\
w\end{array}$ & $\begin{array}{l}22 \\
g\end{array}$ & $\begin{array}{l}23 \\
8\end{array}$ & $\begin{array}{l}24 \\
8\end{array}$ & $\begin{array}{l}25 \\
p\end{array}$ \\
\hline & & & & & & & & & & & & & & \\
\hline \multicolumn{15}{|l|}{$M d h-2$} \\
\hline & $(\mathrm{N})$ & 18 & 11 & 20 & 9 & 3 & 9 & 28 & 9 & 26 & 28 & 25 & 38 & 18 \\
\hline & a & 0.00 & 0.00 & 0.00 & 0.00 & 0.00 & 0.00 & 0.00 & 0.00 & 0.00 & 0.00 & 0.00 & 0.00 & 0.00 \\
\hline & b & 0.00 & 0.00 & 0.00 & 0.00 & 0.00 & 0.00 & 0.00 & 0.00 & 0.00 & 0.00 & 0.00 & 0.00 & 0.00 \\
\hline & c & 1.00 & 1.00 & 1.00 & 1.00 & 1.00 & 1.00 & 1.00 & 1.00 & 1.00 & 1.00 & 1.00 & 1.00 & 1.00 \\
\hline & e & 0.00 & 0.00 & 0.00 & 0.00 & 0.00 & 0.00 & 0.00 & 0.00 & 0.00 & 0.00 & 0.00 & 0.00 & 0.00 \\
\hline & $h$ & - & - & - & - & - & - & - & - & - & - & - & - & - \\
\hline \multicolumn{15}{|l|}{$M e$} \\
\hline & (N) & 26 & 46 & 54 & 47 & 7 & 6 & 37 & 19 & 31 & 25 & 30 & 20 & 11 \\
\hline & b & 0.00 & 0.00 & 1.00 & 1.00 & 1.00 & 1.00 & 1.00 & 1.00 & 1.00 & 0.00 & 0.00 & 0.00 & 0.00 \\
\hline & c & 0.00 & 1.00 & 0.00 & 0.00 & 0.00 & 0.00 & 0.00 & 0.00 & 0.00 & 1.00 & 1.00 & 1.00 & 1.00 \\
\hline & d & 0.00 & 0.00 & 0.00 & 0.00 & 0.00 & 0.00 & 0.00 & 0.00 & 0.00 & 0.00 & 0.00 & 0.00 & 0.00 \\
\hline & e & 1.00 & 0.00 & 0.00 & 0.00 & 0.00 & 0.00 & 0.00 & 0.00 & 0.00 & 0.00 & 0.00 & 0.00 & 0.00 \\
\hline \multicolumn{15}{|l|}{$M p i$} \\
\hline & (N) & 30 & 47 & 43 & $40^{*}$ & 13 & 10 & 24 & 19 & 47 & $48^{*}$ & 18 & 23 & 13 \\
\hline & b & 0.00 & 0.00 & 0.00 & 0.00 & 0.00 & 0.00 & 0.00 & 0.00 & 0.00 & 0.00 & 0.06 & 0.96 & 0.00 \\
\hline & d & 0.00 & 0.00 & 0.00 & 0.00 & 0.00 & 0.00 & 0.00 & 0.00 & 0.00 & 0.84 & 0.80 & 0.04 & 0.92 \\
\hline & e & 0.00 & 0.00 & 0.00 & 0.00 & 0.00 & 0.00 & 0.00 & 0.00 & 0.00 & 0.01 & 0.11 & 0.00 & 0.00 \\
\hline \multirow[t]{4}{*}{ (b) } & f & 0.00 & 0.00 & 0.00 & 0.00 & 0.00 & 0.00 & 0.00 & 0.00 & 0.00 & 0.15 & 0.00 & 0.00 & 0.08 \\
\hline & h & 0.00 & 0.02 & 0.45 & 0.23 & 0.00 & 0.00 & 0.00 & 0.00 & 0.00 & 0.00 & 0.03 & 0.00 & 0.00 \\
\hline & $i$ & 1.00 & 0.00 & 0.00 & 0.00 & 1.00 & 1.00 & 1.00 & 1.00 & 1.00 & 0.00 & 0.00 & 0.00 & 0.00 \\
\hline & $\therefore$ & 0.00 & 0.00 & 0.00 & 0.00 & 0.00 & 0.00 & 0.00 & 0.00 & 0.00 & 0.00 & 0.00 & 0.00 & 0.00 \\
\hline (f) & $\hat{\mathbf{k}}$ & 0.00 & 0.00 & 0.28 & 0.41 & 0.00 & 0.00 & 0.00 & 0.00 & 0.00 & 0.00 & 0.00 & 0.00 & 0.00 \\
\hline \multirow[t]{4}{*}{ (g) } & $\mathbf{m}$ & 0.00 & 0.35 & 0.27 & 0.33 & 0.00 & 0.00 & 0.00 & 0.00 & 0.00 & 0.00 & 0.00 & 0.00 & 0.00 \\
\hline & n & 0.00 & 0.00 & 0.00 & 0.04 & 0.00 & 0.00 & 0.00 & 0.00 & 0.00 & 0.00 & 0.00 & 0.00 & 0.00 \\
\hline & 0 & 0.00 & 0.63 & 0.00 & 0.00 & 0.00 & 0.00 & 0.00 & 0.00 & 0.00 & 0.00 & 0.00 & 0.00 & 0.00 \\
\hline & $h$ & 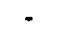 & 0.36 & 0.67 & 0.35 & 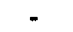 & 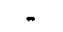 & 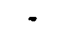 & . & 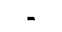 & 0.08 & 0.28 & 0.09 & 0.15 \\
\hline
\end{tabular}

Pep-2

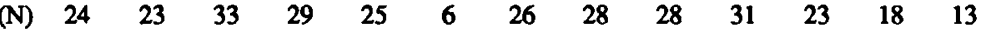

$\begin{array}{llllllllllllll}\text { c } & 0.00 & 0.00 & 0.00 & 0.00 & 0.00 & 0.00 & 0.00 & 0.00 & 0.00 & 0.00 & 0.00 & 0.00 & 1.00\end{array}$

$\begin{array}{llllllllllllll}\text { d } & 0.00 & 0.00 & 0.00 & 0.00 & 0.00 & 0.00 & 0.00 & 0.00 & 0.00 & 0.00 & 0.00 & 1.00 & 0.00\end{array}$

$\begin{array}{lllllllllllllll} & \text { e } & 0.00 & 0.00 & 0.08 & 0.00 & 0.00 & 0.00 & 0.00 & 0.00 & 0.00 & 0.00 & 0.00 & 0.00 & 0.00 \\ \text { (e) } & \text { f } & 0.00 & 0.00 & 0.89 & 0.85 & 0.00 & 0.00 & 0.00 & 0.00 & 0.00 & 0.55 & 0.59 & 0.00 & 0.00\end{array}$

$\begin{array}{llllllllllllll}\text { h } & 0.00 & 0.00 & 0.00 & 0.15 & 0.00 & 0.00 & 0.00 & 0.00 & 0.00 & 0.31 & 0.28 & 0.00 & 0.00\end{array}$

$\begin{array}{llllllllllllll}\text { j } & 1.00 & 0.00 & 0.00 & 0.00 & 0.00 & 0.00 & 0.00 & 0.00 & 0.07 & 0.14 & 0.13 & 0.00 & 0.00\end{array}$

$\begin{array}{llllllllllllll}\text { k } & 0.00 & 0.00 & 0.00 & 0.00 & 0.00 & 0.00 & 0.00 & 0.52 & 0.00 & 0.00 & 0.00 & 0.00 & 0.00\end{array}$

$\begin{array}{llllllllllllll}1 & 0.00 & 1.00 & 0.00 & 0.00 & 0.00 & 0.00 & 0.00 & 0.00 & 0.00 & 0.00 & 0.00 & 0.00 & 0.00\end{array}$

$\begin{array}{llllllllllllll}\mathrm{m} & 0.00 & 0.00 & 0.03 & 0.00 & 1.00 & 1.00 & 1.00 & 0.00 & 0.93 & 0.00 & 0.00 & 0.00 & 0.00\end{array}$

$\begin{array}{llllllllllllll}\mathrm{n} & 0.00 & 0.00 & 0.00 & 0.00 & 0.00 & 0.00 & 0.00 & 0.48 & 0.00 & 0.00 & 0.00 & 0.00 & 0.00\end{array}$

Pep-4

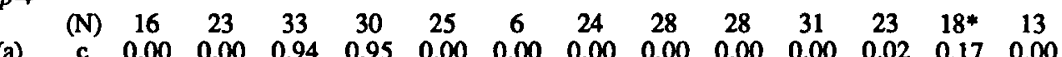
$\begin{array}{llllllllllllll}\text { c } & 0.00 & 0.00 & 0.94 & 0.95 & 0.00 & 0.00 & 0.00 & 0.00 & 0.00 & 0.00 & 0.02 & 0.17 & 0.00 \\ \text { d } & 1.00 & 1.00 & 0.06 & 0.05 & 0.00 & 0.00 & 0.00 & 0.00 & 0.00 & 1.00 & 0.85 & 0.83 & 0.92\end{array}$ $\begin{array}{llllllllllllll}\text { e } & 0.00 & 0.00 & 0.00 & 0.00 & 0.00 & 0.00 & 0.00 & 0.00 & 0.00 & 0.00 & 0.00 & 0.00 & 0.00\end{array}$

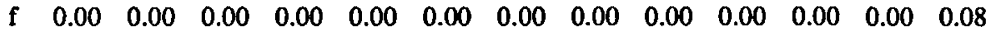
$\begin{array}{llllllllllllll}\mathrm{g} & 0.00 & 0.00 & 0.00 & 0.00 & 0.00 & 0.00 & 0.00 & 0.00 & 0.00 & 0.00 & 0.00 & 0.00 & 0.00\end{array}$ $\begin{array}{llllllllllllll}\mathrm{h} & 0.00 & 0.00 & 0.00 & 0.00 & 1.00 & 1.00 & 1.00 & 1.00 & 1.00 & 0.00 & 0.13 & 0.00 & 0.00\end{array}$

$P k$

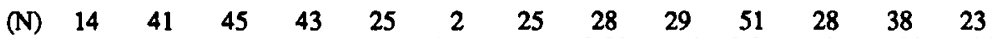
$\begin{array}{llllllllllllll}\text { b } & 0.00 & 0.00 & 0.00 & 0.00 & 0.00 & 0.00 & 0.00 & 0.00 & 0.00 & 0.97 & 1.00 & 1.00 & 1.00\end{array}$

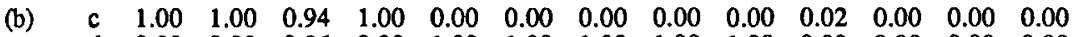
$\begin{array}{llllllllllllll}\text { d } & 0.00 & 0.00 & 0.06 & 0.00 & 1.00 & 1.00 & 1.00 & 1.00 & 1.00 & 0.00 & 0.00 & 0.00 & 0.00\end{array}$ $\begin{array}{llllllllllllll}\text { e } & 0.00 & 0.00 & 0.00 & 0.00 & 0.00 & 0.00 & 0.00 & 0.00 & 0.00 & 0.00 & 0.00 & 0.00 & 0.00\end{array}$

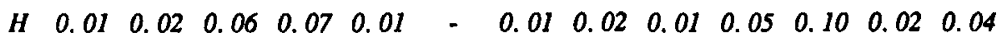


Table V. Number of diagnostic loci (0.99 criterion; Ayala \& Powell, 1972) among species and forms, averaged by area. Data from Scheepmaker et al. (1988) and Scheepmaker \& Van Dalfsen (1989) are included.

\begin{tabular}{|c|c|c|c|c|c|c|c|c|c|c|c|c|c|c|c|c|c|c|}
\hline species & $\begin{array}{c}\text { area(s)/ } \\
\text { station(s) }\end{array}$ & $\begin{array}{l}\text { no. of } \\
\text { samples }\end{array}$ & $\mathbf{J}$ & $\mathrm{j}$ & $\mathbf{M}$ & $\mathbf{L}$ & $\mathrm{B}+\mathrm{C}$ & $\mathbf{F}$ & $\mathrm{I}+17$ & $\mathbf{K}$ & $\mathbf{F}$ & $\mathrm{D}+\mathrm{E}$ & $\mathrm{H}$ & G & I & $\mathrm{B}+\mathrm{C}$ & $\begin{array}{c}\mathrm{J}+\mathrm{L} \\
25, \mathrm{j}, \mathrm{t}\end{array}$ & $\mathbf{H}+\mathbf{H}$ \\
\hline $\begin{array}{c}\text { G. fossarum } \\
\text { s. } l .\end{array}$ & J & 5 & - & & & & & & & & & & & & & & & \\
\hline G. fossarum & $\mathbf{j}$ & 1 & 2 & - & & & & & & & & & & & & & & \\
\hline G. fossarum & $\mathbf{M}$ & 3 & 4 & $2^{\circ}$ & - & & & & & & & & & & & & & \\
\hline $\begin{array}{c}\text { s. str. } \\
\text { G. fossarum } \\
\text { s. } l .\end{array}$ & $\mathbf{L}$ & 3 & 1 & - & 2 & - & & & & & & & & & & & & \\
\hline $\begin{array}{c}\text { G. fossarum } \\
\text { s. } l \text {. }\end{array}$ & $\mathrm{B}+\mathrm{C}$ & 6 & - & 2 & 4 & - & - & & & & & & & & & & & \\
\hline G. fossarum & $\mathbf{F}$ & 2 & 1 & 3 & 4 & 1 & 1 & - & & & & & & & & & & \\
\hline $\begin{array}{c}\text { G. fossarum } \\
\text { s. } l .\end{array}$ & $\mathrm{I}+17$ & 2 & 1 & 3 & 4 & - & - & 1 & - & & & . & & & & & & \\
\hline $\begin{array}{c}\text { G. fossarum } \\
\text { s. } l .\end{array}$ & $\mathbf{K}$ & 2 & - & 2 & 2 & - & - & 1 & - & - & & & & & & & & \\
\hline G. orinos & F & 3 & 3 & 2 & 4 & 2 & 4 & 4 & 2 & 2 & - & & & & & & & \\
\hline G. orinos & D+E & 4 & - & - & 2 & - & - & - & - & - & - & - & & & & & & \\
\hline G. cf. fossarum & $\mathbf{H}$ & 3 & 5 & 4 & 5 & 3 & 5 & 4 & 4 & 5 & 4 & 1 & - & & & & & \\
\hline G. cf. fossarum & G & 2 & 4 & 5 & 6 & 3 & 5 & 5 & 4 & 4 & 4 & 2 & 4 & - & & & & \\
\hline G. stupendus & I & 3 & 1 & - & 2 & - & 2 & 3 & 2 & 2 & - & - & 3 & 3 & - & & & \\
\hline G. wautieri & $\mathrm{B}+\mathrm{C}$ & 5 & 6 & 7 & 4 & 3 & 6 & 3 & 6 & 6 & 6 & 4 & 5 & 7 & 5 & - & & \\
\hline G. p.pulex & $\begin{array}{l}\mathrm{J}+\mathrm{L}, \\
25, \mathrm{j}, \mathrm{t}\end{array}$ & 5 & 3 & 4 & 5 & 4 & 5 & 6 & 5 & 4 & 4 & 3 & 7 & 4 & 4 & 6 & - & \\
\hline G.p.gallicus & $\mathrm{I}+\mathrm{H}$ & 3 & 2 & 4 & 4 & 2 & 2 & 4 & 3 & 2 & - & 3 & 5 & 3 & 2 & 5 & 1 & - \\
\hline
\end{tabular}

maker et al., 1988 and Scheepmaker \& Van Dalfsen, 1989; Table I, stations $\mathrm{a}-\mathrm{t}$ ) are summarized in Table V. Values of $I$ and $D$, averaged by taxon, and by area, are listed in Tables VI and VII, respectively. Because of the low levels of divergence recorded, and the considerable geographic distances separating most sampling localities (Table I; Fig. 1A), G. p. pulex and G. p. gallicus were averaged by form, not by area. In the UPGMA dendrogram (Fig. 2) and the Wagner distance network (Fig. 3), clearly distinct clusters are formed by $G$. fossarum s.str., G. stupendus, G. wautieri and G. p. pulex + G. p. gallicus. Intra-specific inter-areal divergence may be considerable: inter-areal $I$-values among $G$. orinos from areas $\mathrm{D} / \mathrm{E}$, and $\mathrm{F}$ range from $0.67-0.87$ $(D=0.40-0.14)$; among $G$. cf. fossarum from areas $\mathrm{G}$ and $\mathrm{H}$ inter-areal $I$-values range from
$0.63-0.69(D=0.47-0.38)$. As a consequence, neither the conspecific $G$. orinos samples from areas D/E, and F, nor the conspecific G. cf. fossarum samples from areas $\mathrm{G}$ and $\mathrm{H}$ form monophyletic groups in Figs. 2 and 3.

Inter-specific inter-areal $I$-values among $G$. orinos and $G$. cf. fossarum do not differ significantly from intra-specific inter-areal values in a MannWhittney U-test at the 0.05 level (except for G. orinos from areas D, E and F, vs. G. cf. fossarum from area $\mathrm{H}$ and $G$. orinos from area $\mathrm{F}$ ). Moreover, inter-areal $I$-values between any of the combinations $\mathrm{F}, \mathrm{D} / \mathrm{E}, \mathrm{G}$ and $\mathrm{H}$ do not differ significantly at the 0.05 level. To put it another way, $G$. orinos from areas D/E seems to differ about as much from $G$. orinos from area $\mathrm{F}$, as it does from $G$. cf. fossarum from area $\mathrm{G}$, or $\mathrm{H}$, and vice versa. The same 


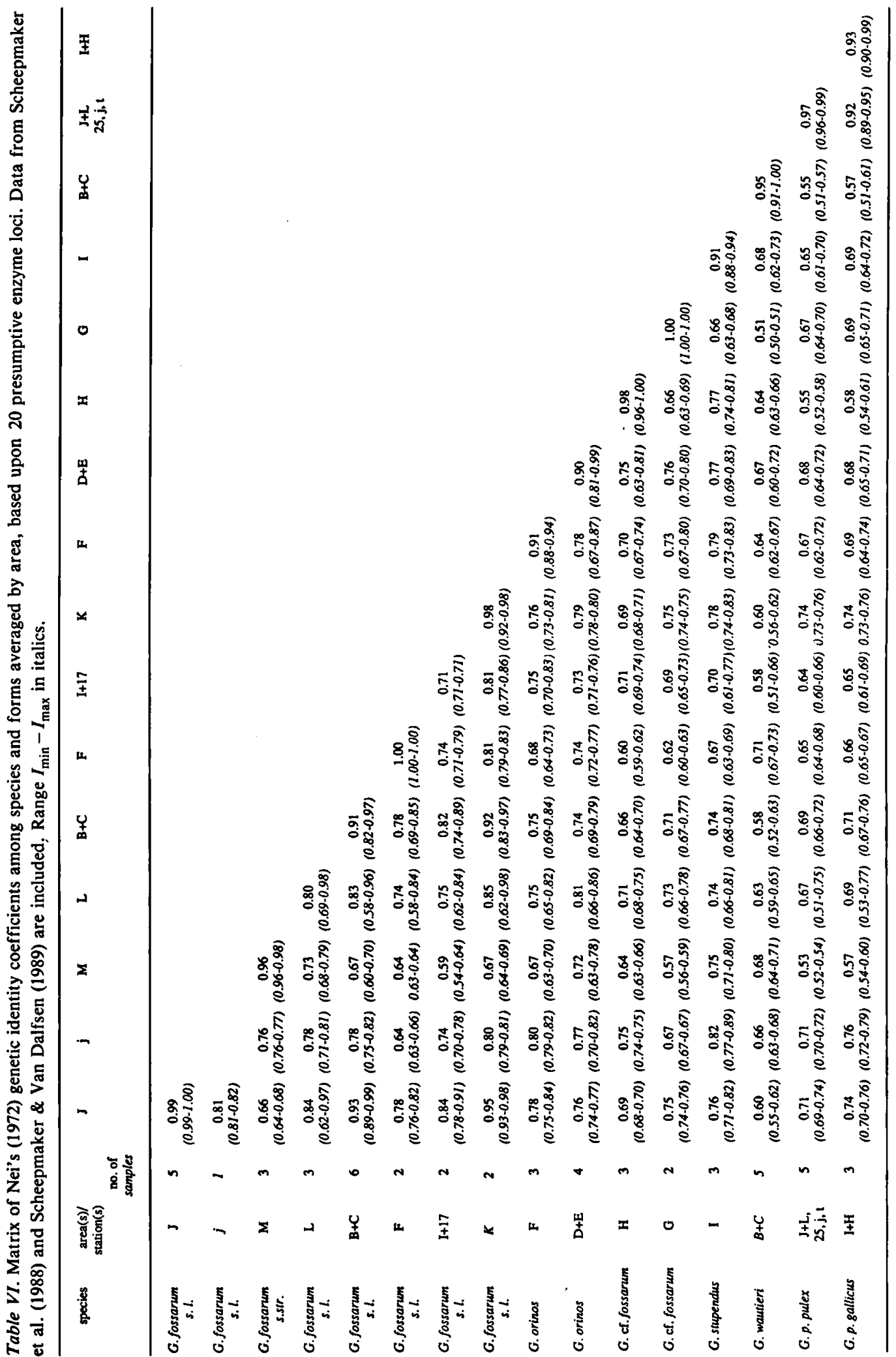




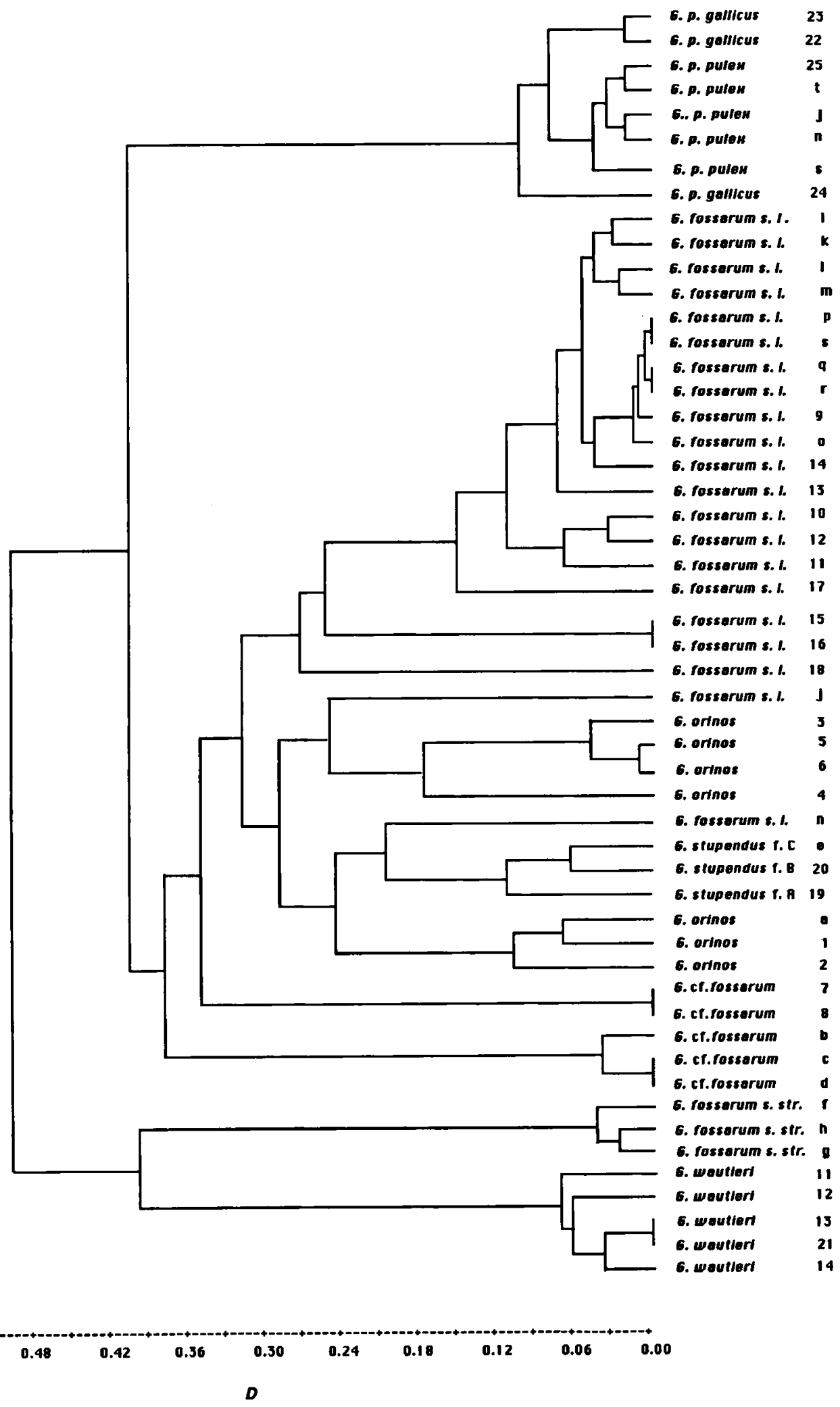

Fig. 2. UPGMA dendrogram of Nei's (1972) genetic distance $(D)$ based upon 20 enzyme loci. 


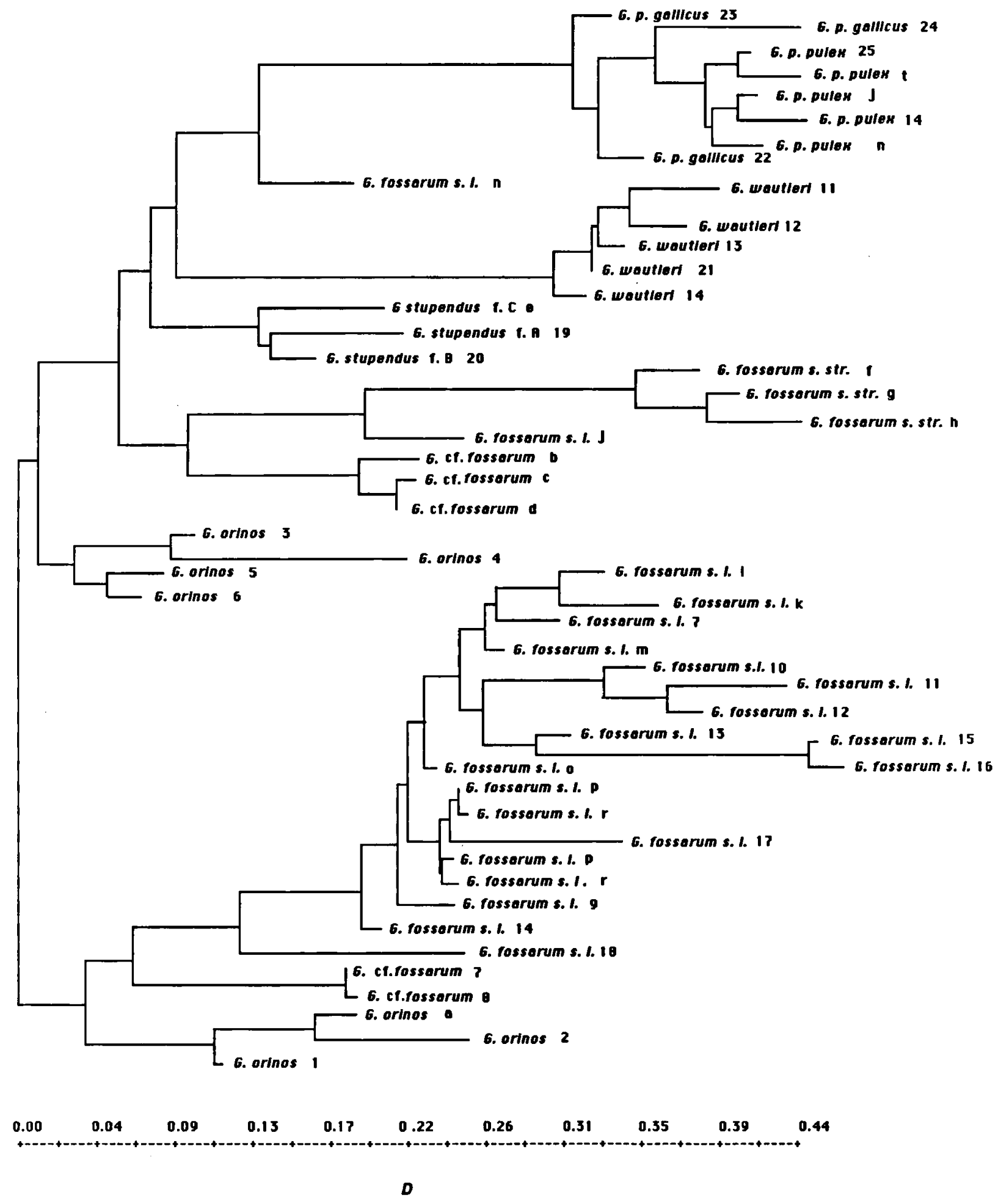

Fig. 3. Distance Wagner network of Rogers' (1972) genetic distance rooted at the midpoint of the longest path (Farris, 1972) based upon 20 enzyme loci. 


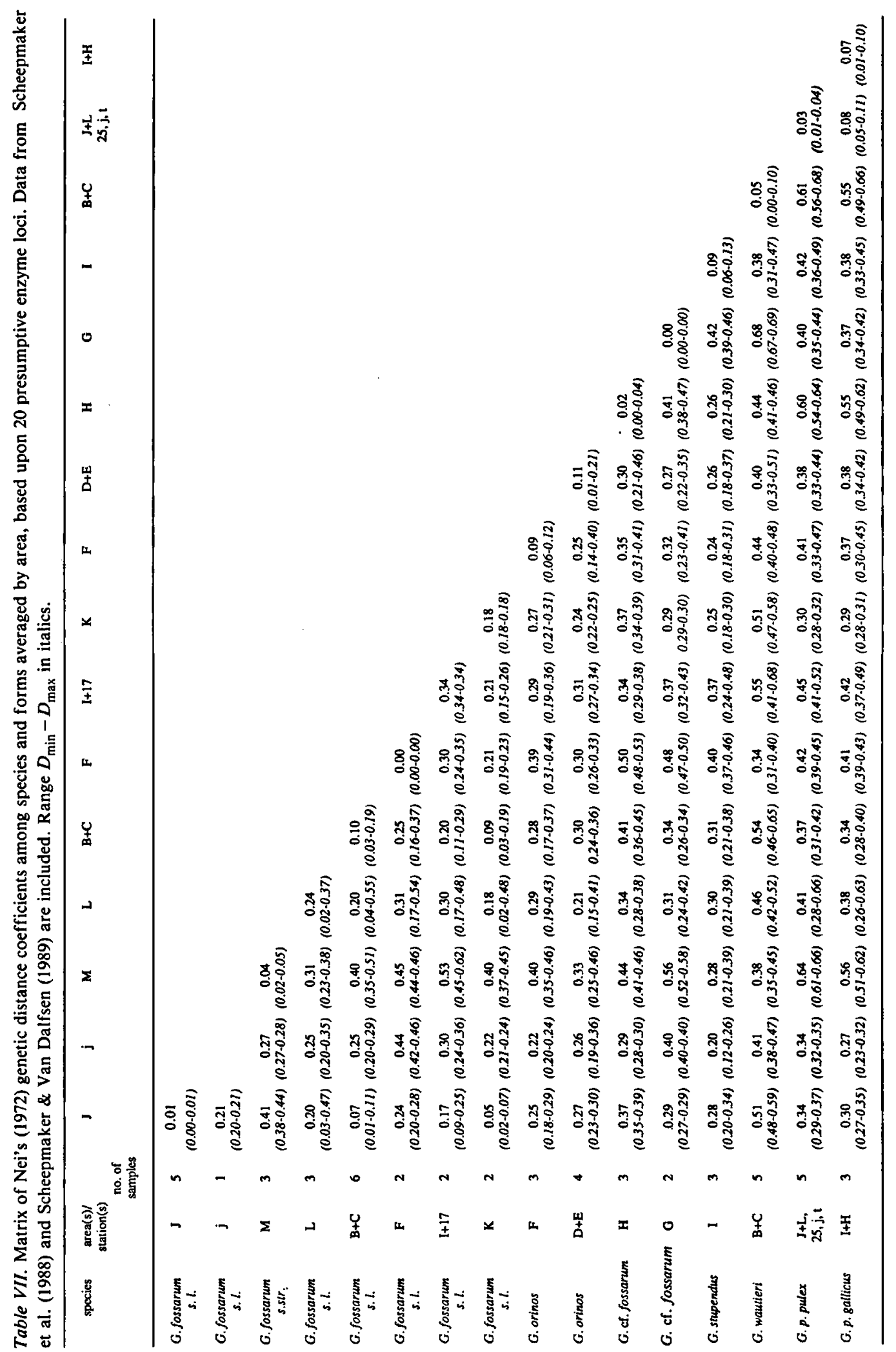


Table VIII. $\theta$-indices according to Weir \& Cockerham (1984) of the species examined. Data from Scheepmaker et al. (1988), and Scheepmaker \& Van Dalfsen (1989) are included. G. foss. s.l. = G. fossarum s.l.; G. foss. s. str. = G. fossarum s. str.; G. or. = G. orinos; G. cf. foss. = G. cf. fossarum; G. stup. = G. stupendus; G. waut. = G. wautieri; G. p.p. = G. p. pulex; G. p. g. = G. p. gallicus. Significance of population differentiation was tested with a chi-square heterogeneity test; ${ }^{*}<<0.05$.

\begin{tabular}{|c|c|c|c|c|c|c|c|c|}
\hline locus & G. foss. s. $l$. & G. foss. s. str. & G. or. & G. cf. foss. & G. stup. & G. waut. & G.p.p. & G. p. 8 . \\
\hline Apk & - & $0.15^{*}$ & - & - & - & - & - & - \\
\hline Est-I & - & $0.36^{*}$ & - & $1.00 *$ & - & - & - & - \\
\hline Est-2 & $0.63^{*}$ & - & - & - & 0.01 & - & - & - \\
\hline Got-l & $0.56 *$ & $0.04^{*}$ & $0.90^{*}$ & $1.00 *$ & $0.89^{*}$ & 0.02 & - & $0.11^{*}$ \\
\hline Got-2 & $0.77^{*}$ & $0.25^{*}$ & - & - & - & - & 0.00 & - \\
\hline Gpi & $0.57^{*}$ & $0.20 *$ & $0.58^{*}$ & $0.50^{*}$ & $0.83^{*}$ & $0.22 *$ & $0.06^{*}$ & $0.28^{*}$ \\
\hline$H k$ & $1.00^{*}$ & - & - & - & - & - & - & - \\
\hline$M d h-1$ & $0.30^{*}$ & $0.06^{*}$ & $0.72 *$ & - & $0.07^{*}$ & $0.63^{*}$ & $0.39 *$ & $0.07^{*}$ \\
\hline$M d h-2$ & 0.00 & 0.01 & 0.01 & $0.40^{*}$ & - & - & - & - \\
\hline$M e$ & $1.00^{*}$ & - & $1.00^{*}$ & - & - & - & - & - \\
\hline$M p i$ & $0.59 *$ & $0.46^{*}$ & $0.72^{*}$ & $0.96^{*}$ & $0.40^{*}$ & - & $0.49^{*}$ & $0.67^{*}$ \\
\hline Pep-2 & $0.73^{*}$ & $0.05^{*}$ & $0.76^{*}$ & $1.00^{*}$ & $0.15^{*}$ & $0.68 *$ & 0.02 & $0.47^{*}$ \\
\hline Pep-4 & $0.34^{*}$ & - & $0.05^{*}$ & $0.97^{*}$ & $0.05^{*}$ & - & $0.38^{*}$ & $0.10^{*}$ \\
\hline$P k$ & $0.13^{*}$ & - & $1.00^{*}$ & - & 0.06 & - & - & - \\
\hline Mean & 0.63 & 0.20 & 0.75 & 0.86 & 0.67 & 0.59 & 0.38 & 0.42 \\
\hline
\end{tabular}

Table IX. $\theta$-indices according to Weir \& Cockerham (1984) of subsets of the species examined. For legend see Table VIII.

\begin{tabular}{|c|c|c|c|c|c|c|c|c|c|c|}
\hline $\begin{array}{l}\text { Area(s) } \\
\text { Stations }\end{array}$ & $\begin{array}{c}\text { G. foss. s.l. } \\
\text { B, C, J, L } \\
9-14 ; \mathrm{k}-\mathrm{m} ; \\
\text { o-s }\end{array}$ & $\begin{array}{l}\text { foss. s.l } \\
\text { B, C } \\
9-14\end{array}$ & $\begin{array}{l}\text { J. foss. s.l } \\
\text { C } \\
9-12 ; 14\end{array}$ & $\underset{\mathrm{J}}{\text { foss. } s . \mathrm{s}}$ & $\begin{array}{c}\text { foss. s.l } \\
\mathbf{J} \\
\mathbf{p}-\mathbf{s}\end{array}$ & $\begin{array}{l}\text { foss. s.l. } \\
\text { K, L } \\
\text { i; k-m }\end{array}$ & $\begin{array}{c}\text { G. or. } \\
\text { D, E } \\
3-6\end{array}$ & $\begin{array}{l}\text { G. or. } \\
\underset{\mathbf{F}}{\text { a: }} 1-2\end{array}$ & $\begin{array}{c}\text { G. cf. foss. } \\
\text { b-d }\end{array}$ & $\begin{array}{c}\text { G. waut. } \\
\text { B, C } \\
12-13 ; 15\end{array}$ \\
\hline \multicolumn{11}{|l|}{ locus } \\
\hline Est-2 & $0.87^{*}$ & $0.59 *$ & $0.53^{*}$ & - & - & - & $0.25^{*}$ & - & - & - \\
\hline Got-1 & $0.25^{*}$ & $0.17^{*}$ & $0.20^{*}$ & 0.00 & 0.01 & $0.22 *$ & 0.03 & $0.52^{*}$ & - & 0.01 \\
\hline Got-2 & $0.76^{*}$ & $0.98^{*}$ & $0.98^{*}$ & 0.04 & 0.04 & $0.45^{*}$ & - & - & - & - \\
\hline$G p i$ & $0.53^{*}$ & $0.40^{*}$ & $0.42^{*}$ & $0.35^{*}$ & 0.00 & $0.16^{*}$ & $0.27^{*}$ & $0.12^{*}$ & $0.49^{*}$ & 0.01 \\
\hline$M d h-1$ & $0.19^{*}$ & 0.00 & 0.00 & 0.00 & 0.00 & $0.14^{*}$ & $0.80^{*}$ & $0.28^{*}$ & - & 0.06 \\
\hline$M d h-2$ & 0.01 & 0.01 & 0.01 & - & - & 0.01 & . & - & $0.06^{*}$ & - \\
\hline$M p i$ & $0.59^{*}$ & $0.49^{*}$ & $0.50^{*}$ & $0.15^{*}$ & $0.19^{*}$ & $0.10^{*}$ & $0.47^{*}$ & - & 0.00 & - \\
\hline Pep-2 & $0.50^{*}$ & $0.48^{*}$ & $0.48^{*}$ & 0.02 & 0.05 & - & $0.60^{*}$ & $1.00^{*}$ & 0.02 & 0.03 \\
\hline Pep-4 & $0.36^{*}$ & $0.26^{*}$ & $0.29^{*}$ & 0.00 & 0.00 & 0.25 & $0.05^{* *}$ & 0.01 & $0.38^{*}$ & - \\
\hline$P k$ & $0.13^{*}$ & - & - & - & - & - & - & - & - & - \\
\hline Mean & 0.48 & 0.59 & 0.53 & 0.18 & 0.12 & 0.25 & 0.60 & 0.56 & 0.31 & 0.03 \\
\hline
\end{tabular}

holds true for each of these subsets with regard to G. stupendus and G. fossarum s.l.. Although interareal $I$-values of this combination are not statistically significant in a Mann-Whittney U-test, the highest intra-specific inter-areal value of $I(I=0.87)$ was found among $G$. orinos from areas $\mathrm{D}, \mathrm{E}$ and $\mathrm{F}$.

G. fossarum s.l. from areas I, F, B/C, L, K and $\mathrm{J}$, plus stations 17 and $\mathrm{O}$ (Fig. 1A, Table I), although genetically well differentiated $\left(I_{\min }-I_{\max }\right.$ $=0.69-0.99)$, forms a delimited monophyletic 
Table X. $\theta$-indices according to Weir \& Cockerham (1984) of pairwise combinations of samples of the species examined. For legend see Table VIII.

\begin{tabular}{|c|c|c|c|c|c|c|c|c|c|c|c|}
\hline $\begin{array}{l}\text { Area(s) } \\
\text { Stations }\end{array}$ & $\begin{array}{c}\text { G. foss. s.l. } \\
\underset{10,12}{C}\end{array}$ & $\begin{array}{l}\text { foss. s.l } \\
\text { C, } 14\end{array}$ & $\begin{array}{c}\text { G. foss. s.l. } \\
\mathbf{K} \\
1, \mathbf{m}\end{array}$ & $\begin{array}{c}\text { G. foss. s.l } \\
\mathrm{L} \\
\mathrm{i}, \mathrm{k}\end{array}$ & $\begin{array}{c}\text { G. foss. s.l. } \\
\text { F } \\
15,16\end{array}$ & $\begin{array}{c}\text { G. foss. s.l. } \\
I 7,18\end{array}$ & $\begin{array}{c}\text { G. or. } \\
\text { D, E } \\
5,6\end{array}$ & $\begin{array}{c}\text { G. or. } \\
\mathbf{F} \\
\mathbf{a} ; 1\end{array}$ & $\begin{array}{c}\text { G. cf. foss. } \\
\mathbf{H} \\
\mathrm{c}, \mathrm{d}\end{array}$ & $\begin{array}{c}\text { G. cf. foss. } \\
\mathbf{G} \\
7,8\end{array}$ & $\begin{array}{c}\text { G. p. } \\
\underset{G}{G} . \\
22,23\end{array}$ \\
\hline \multicolumn{12}{|l|}{ locus } \\
\hline Est-I & - & - & - & - & - & - & $0.25^{*}$ & - & - & - & - \\
\hline Est-2 & $0.22 *$ & - & - & . & 0.07 & $1.00^{*}$ & 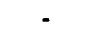 & - & - & - & - \\
\hline Got-1 & 0.01 & $0.20^{*}$ & $0.05^{*}$ & $0.13^{*}$ & $0.07^{*}$ & - & 0.03 & 0.01 & - & - & 0.11 \\
\hline Got-2 & 0.00 & - & $0.02 *$ & $0.38 *$ & 0.01 & - & $\cdot$ & - & - & - & - \\
\hline$G p i$ & $0.06^{*}$ & $0.54^{*}$ & 0.00 & $0.05^{*}$ & 0.00 & $0.90^{*}$ & 0.01 & 0.00 & 0.01 & - & 0.20 \\
\hline Mdh-1 & 0.02 & 0.00 & $0.12^{*}$ & $0.08^{*}$ & 0.03 & $1.00^{*}$ & $0.07^{*}$ & $0.42^{*}$ & - & - & 0.07 \\
\hline$M d h-2$ & - & 0.01 & 0.00 & - & 0.00 & - & - & 0.03 & 0.04 & - & - \\
\hline$M p i$ & $0.41 *$ & $0.41^{*}$ & $0.06 *$ & $0.12 *$ & 0.01 & $0.72 *$ & - & - & - & 0.03 & 0.03 \\
\hline Pep-2 & 0.07 & $0.13^{*}$ & - & - & - & $1.00^{*}$ & 0.04 & $1.00^{*}$ & - & & 0.02 \\
\hline Pep-4 & $0.23^{*}$ & - & $0.49 *$ & $0.05^{*}$ & 0.02 & - & 0.01 & 0.01 & - & 0.03 & 0.14 \\
\hline$P k$ & - & & - & - & - & - & - & - & - & - & 0.14 \\
\hline Mean & 0.25 & 0.35 & 0.29 & 0.15 & 0.04 & 0.93 & 0.05 & 0.61 & 0.02 & 0.03 & 0.09 \\
\hline
\end{tabular}

group of genetically related forms in Figs. 2 and 3. The recorded variation is not always congruent with the geographic distribution and appears to be gradual; sharp discontinuities are often lacking. However, due to increased local variation, population samples from areas B/C in Figs. 2 and 3 do not cluster together and they are occasionally closer to population samples from other areas (e.g. G. fossarum s.l. from stations 9 and 14 with $G$. fossarum s.l. from area J). G. fossarum s.l. from stations 17 and 18 (area I) are genetically rather distant and cluster apart in Figs. 2 and 3. However, G. fossarum s.l. from stations $\mathrm{j}$ and $\mathrm{n}$ deviate strongly from this pattern; in Fig. 2 they cluster close to $G$. stupendus and G. orinos, and in Fig. 3 to G. pulex and to $G$. fossarum s. str., respectively.

\section{Genetic structure of populations and levels of gene flow}

The results of the $F$-statistic analysis $(\theta)$ for $G$. fossarum s.l., G. p. pulex, G. p. gallicus, G. wautieri, $G$. orinos, $G$. stupendus and $G$. cf. fossarum are shown in Table VIII. In the following procedures, because of their strongly deviating character, $G$. fossarum s.l. from stations $\mathrm{j}$ and $\mathrm{n}$ have been omitted. Significant population subdivision was found for all species at a high number of polymorphic loci (e.g. all 7 in G. cf. fossarum; 8 of 9 in G. orinos; 11 of 12 in $G$. fossarum s.l.). Creating subsets by omitting genetically deviating samples or by combining stations by area (Table IX) does not change this pattern in most cases; however, much less population subdivision was found in subsets formed by $G$. fossarum s.l. ( 2 of 7 loci in stations $0-\mathrm{s}$, and 1 of 7 in stations $\mathrm{p}-\mathrm{s}$ ) and $G$. wautieri (none in stations 12,13 and 15). Subsets formed by pairwise combinations of genetically similar and geographically close populations (Table X) show that within several species and areas statistically significant population subdivision still exists over short distances (e.g. stations $i-k$, all 6 loci; stations 1-m, 5 of 7 loci; stations $10-12$ and 9-14, 4 of 8, and 4 of 6 loci, respectively).

Mean values of $\theta$ and $F_{S T}$ within species and subsets of population samples are listed in Tables $\mathrm{XI}-\mathrm{XIII}$, along with the corresponding $\mathrm{Nm}$ values. For sets of 4 population samples and more, the average frequencies of private alleles $\bar{p}(1)$ and the corresponding $\mathrm{Nm}$ - and $\mathrm{Nm}^{*}$-values figure in Tables XI-XII. Values of $\theta$ are generally higher than those of $F_{S T}$. Consequently, levels of gene flow derived from $\theta$ are lower than those derived from $F_{S T}$. Genetic drift and bottlenecks will result 
Table XI. Various Nm estimates in G. fossarum s.l., G. fossarum s. str., G. wautieri, G. stupendus, G. orinos, G. cf. fossarum, G. p. pulex and G. p. gallicus. $\bar{p}(1)=$ average frequency of private alleles (Slatkin, 1985); $\mathrm{Nav}=$ average sample size; $\mathrm{Nm}=$ estimate derived from $\bar{p}(1) ; N m^{*}=$ idem, with correction for sample size; $F_{S T}=F$-statistic (Wright, 1931); $\theta=$ idem (Weir \& Cockerham, 1984); $N m_{F_{S T}}=N m$ estimate derived from $F_{S T} ; N m_{\theta}=$ idem, derived from $\theta ;$ n.p.a. = number of private alleles; $\mathrm{km}(\mathrm{av})=$ average geographic distance among samples in kilometres.

\begin{tabular}{|c|c|c|c|c|c|c|c|c|c|c|c|c|c|}
\hline Species & Area(s) & Stations & $\begin{array}{l}\text { No. of } \\
\text { samples }\end{array}$ & km (av) & $F_{S T}$ & $\theta$ & n.p.a & $\bar{p}(1)$ & $\mathrm{Nav}$ & $\mathrm{Nm}$ & $N m^{*}$ & $N m F_{S T}$ & $N m_{\theta}$ \\
\hline $\begin{array}{l}\text { G. fossarum s.l. } \\
\text { G. fossarum s. str. } \\
\text { G. wautieri } \\
\text { G. stupendus } \\
\text { G. orinos } \\
\text { G. cf. fossarum } \\
\text { G. p. pulex } \\
\text { G.p. gallicus }\end{array}$ & $\begin{array}{c}\text { B, C, F, I-L } \\
\text { M } \\
\text { B,C } \\
\text { I } \\
\text { D, E, F } \\
\text { H, G } \\
\text { J, L } \\
\text { G, I }\end{array}$ & $\begin{array}{c}9-18 ; j-m ; o-s \\
\text { f-h } \\
11-14 ; 21 \\
19,20 ; \mathrm{e} \\
1-6 ; \mathrm{a} \\
7,8 ; \text { b-d } \\
25 ; \mathrm{j}, \mathrm{n}, \mathrm{s}, \mathrm{t} \\
22-24\end{array}$ & $\begin{array}{l}19 \\
3 \\
5 \\
3 \\
7 \\
5 \\
5 \\
3\end{array}$ & $\begin{array}{l}306 \\
25 \\
87 \\
21 \\
93 \\
39 \\
352 \\
169\end{array}$ & $\begin{array}{l}0.68 \\
0.16 \\
0.74 \\
0.47 \\
0.71 \\
0.86 \\
0.36 \\
0.35\end{array}$ & $\begin{array}{l}0.63 \\
0.20 \\
0.59 \\
0.67 \\
0.75 \\
0.86 \\
0.38 \\
0.42\end{array}$ & $\begin{array}{l}12 \\
6 \\
7 \\
6 \\
8\end{array}$ & $\begin{array}{l}0.19 \\
\\
0.36 \\
\\
0.35 \\
0.18 \\
0.11\end{array}$ & $\begin{array}{l}33.31 \\
13.03 \\
33.93 \\
37.65 \\
21.06\end{array}$ & $\begin{array}{l}0.21 \\
\\
0.06 \\
\\
0.06 \\
0.25 \\
0.64\end{array}$ & $\begin{array}{l}0.16 \\
0.11 \\
0.05 \\
0.16 \\
0.76\end{array}$ & $\begin{array}{l}0.12 \\
1.31 \\
0.09 \\
0.28 \\
0.10 \\
0.04 \\
0.44 \\
0.46\end{array}$ & $\begin{array}{l}0.15 \\
0.99 \\
0.18 \\
0.13 \\
0.08 \\
0.04 \\
0.40 \\
0.35\end{array}$ \\
\hline
\end{tabular}

Table XII. Various Nm-estimates in subsets of G. fossarum s.l., G. orinos, G. cf. fossarum, and G. p. pulex. For legend see Table XI.

\begin{tabular}{|c|c|c|c|c|c|c|c|c|c|c|c|c|c|}
\hline Species & Area(s) & Stations & $\begin{array}{r}\text { No. of } \\
\text { samples }\end{array}$ & km (av) & $F_{S T}$ & $\theta$ & n.p.a & $\bar{p}(1)$ & Nav & $N m$ & $N m^{*}$ & $N m F_{S T}$ & $N m_{\theta}$ \\
\hline G. fossarum s.l. & B, C,J-L & $9-14 ; i, k-m ; o-s$ & 15 & 289 & 0.50 & 0.48 & 8 & 0.03 & 34.89 & 8.26 & 5.92 & 0.25 & 0.27 \\
\hline G. fossarum s. l. & B, C & $9-14$ & 6 & 35 & 0.55 & 0.59 & 5 & 0.12 & 29.94 & 0.50 & 0.42 & 0.20 & 0.17 \\
\hline G. fossarum s. l. & $\mathrm{B}, \mathrm{C}$ & $9-12 ; 14$ & 5 & 12 & 0.50 & 0.53 & & & & & & 0.26 & 0.22 \\
\hline G. fossarum s. $l$. & $\mathbf{J}$ & $0-s$ & 5 & 37 & 0.15 & 0.18 & 5 & 0.06 & 32.53 & 1.96 & 1.51 & 1.41 & 1.17 \\
\hline G. fossarum s.l. & $\mathbf{J}$ & p-s & 4 & 8 & 0.10 & 0.12 & & & & & & 2.20 & 1.75 \\
\hline G. fossarum s. $l$. & $\mathrm{K}, \mathrm{L}$ & $\mathrm{i}, \mathrm{k}-\mathrm{m}$ & 4 & 270 & 0.21 & 0.25 & 9 & 0.06 & 45.58 & 2.03 & 1.11 & 0.93 & 0.75 \\
\hline G.orinos & $\mathrm{D}, \mathrm{E}$ & $3-6$ & 4 & 35 & 0.53 & 0.60 & 6 & 0.25 & 34.10 & 0.12 & 0.09 & 0.22 & 0.17 \\
\hline G. orinos & $\mathbf{F}$ & 1,$2 ; \mathbf{a}$ & 3 & 9 & 0.55 & 0.56 & & & & & & 0.20 & 0.19 \\
\hline G.cf. fossarum & $\mathbf{H}$ & b-d & 3 & 13 & 0.27 & 0.31 & & & & & & 0.68 & 0.56 \\
\hline G. p. pulex & $\mathbf{J}, \mathbf{L}$ & $25 ; \mathrm{n}, \mathrm{s}, \mathrm{t}$ & 4 & 292 & 0.32 & 0.38 & & & & & & 0.53 & 0.40 \\
\hline G. wautieri & B, C & 12,$13 ; 15$ & 3 & 35 & 0.02 & 0.03 & & & & & & 12.25 & 8.08 \\
\hline
\end{tabular}

Table XIII. Nm estimates derived from $F_{S T}$ (Wright, 1931) and $\theta$ (Weir and Cockerham, 1984) in pairwise combinations of G. fossarum s.l., G. orinos, G. cf. fossarum, and G. p. gallicus. For legend see Table VIII.

\begin{tabular}{|c|c|c|c|c|c|c|c|}
\hline Species & Area(s) & Stations & km (av) & $F_{S T}$ & $\theta$ & $N m F_{S T}$ & $N m_{\theta}$ \\
\hline $\begin{array}{l}\text { G. fossarum s. } l \text {. } \\
\text { G. fossarum s. } l \text {. } \\
\text { G. fossarum s. } \\
\text { G. fossarum s. } l \text {. } \\
\text { G. fossarum s. } l \text {. } \\
\text { G. fossarum s. } l \text {. } \\
\text { G.orinos } \\
\text { G.orinos } \\
\text { G. cf. fossarum } \\
\text { G. cf. fossarum } \\
\text { G.p. gallicus }\end{array}$ & $\begin{array}{c}\mathbf{C} \\
\mathbf{C} \\
\mathbf{I} \\
\mathbf{F} \\
\mathbf{K} \\
\mathbf{L} \\
\mathbf{D}, \mathbf{E} \\
\mathbf{F} \\
\mathbf{H} \\
\mathbf{G} \\
\mathbf{G}\end{array}$ & $\begin{array}{c}10,12 \\
9,14 \\
17,18 \\
15,16 \\
\text { l,m } \\
\text { i,k } \\
5,6 \\
1 ; \mathrm{a} \\
c, d \\
7,8 \\
22,23\end{array}$ & $\begin{array}{c}5 \\
4 \\
55 \\
3 \\
40 \\
41 \\
65 \\
3 \\
10 \\
4 \\
7\end{array}$ & $\begin{array}{l}0.15 \\
0.23 \\
0.89 \\
0.03 \\
0.19 \\
0.08 \\
0.03 \\
0.42 \\
0.02 \\
0.02 \\
0.06\end{array}$ & $\begin{array}{l}0.25 \\
0.35 \\
0.93 \\
0.04 \\
0.29 \\
0.15 \\
0.05 \\
0.61 \\
0.02 \\
0.03 \\
0.09\end{array}$ & $\begin{array}{c}1.39 \\
0.86 \\
0.03 \\
7.10 \\
1.05 \\
2.91 \\
7.10 \\
0.35 \\
15.38- \\
10.62- \\
4.21\end{array}$ & $\begin{array}{r}0.74 \\
0.47 \\
0.02 \\
5.56 \\
0.63 \\
1.42 \\
4.79 \\
0.16 \\
11.38 \\
8.51 \\
2.53\end{array}$ \\
\hline
\end{tabular}

in substantial local differentiation if $\mathrm{Nm}<1$; when $N m>1$, populations tend to become panmictic for neutral alleles (Wright, 1931). In most species and subsets examined, $N m^{*}, N m_{F_{s T}}$, and $N m_{\theta}$ are $<1$ (Tables XI-XIII), and these values are much lower than expected based on dispersal abilities in a stable aquatic environment. At first sight, the important geographic distances separating some of the sampling localities of populations forming sets and subsets in Tables XI-XIII are likely to account for the low $N m$-values observed. However, based upon geographic distance data in Tables XI-XIII, there does not seem to be a correlation with $N m_{\theta}$-values. 

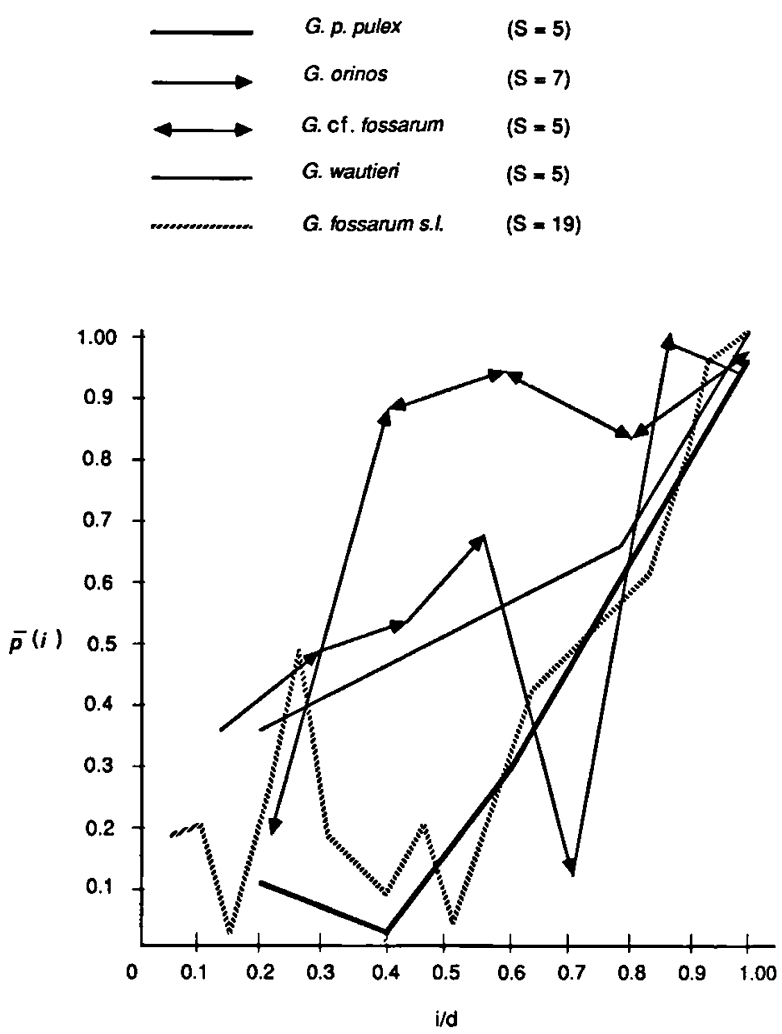

Fig. 4. CAF gene flow profiles (Slatkin, 1981) in G. p. pulex, G. orinos, G. cf. fossarum, G. wautieri and G. fossarum s.l. $\mathrm{S}=$ number of samples; $\bar{p}(i)=$ conditional average frequency (CAF) of an allele; $i / d=$ incidence of an allele, where $i=$ the total number of samples it appears in, and $d=$ the total number of samples examined. The points plotted are average values of $p(i)$ for all alleles having the same $i / d$ value.

Slatkin's (1985) approach of private alleles $\left(\mathrm{Nm}^{*}\right)$ has been applied to 10 sets of populations. Only once (Table XII: G. fossarum s.l., areas B, C, $\mathrm{J}-\mathrm{L}$, stations 9-14; $\mathrm{i}, \mathrm{k}-\mathrm{m}, \mathrm{o}-\mathrm{s}$ ) this method yielded an estimate that was in contrast with the $\mathrm{Nm}_{\theta}$ estimate; in 4 cases (Table XI: G. fossarum s.l., G. wautieri and G. orinos; Table XII: G. fossarum s.l., area J, stations o-s) a close agreement of values of $\mathrm{Nm}^{*}$ and $\mathrm{Nm}_{\theta}$ was found. Although both $N m_{\theta}$ and $N m^{*}$-values are generally low within all species and most of the species subsets sampled by area, subsampling shows that there are possibly high levels of gene flow among many populations. In Table XII this is illustrated by $G$. wautieri (areas B and C, stations $12-13,15$ ) and $G$. fossarum s.l. (area J, stations $\mathrm{o}-\mathrm{s}$ and $\mathrm{p}-\mathrm{s}$ ) yielding $N m_{\theta}$-values
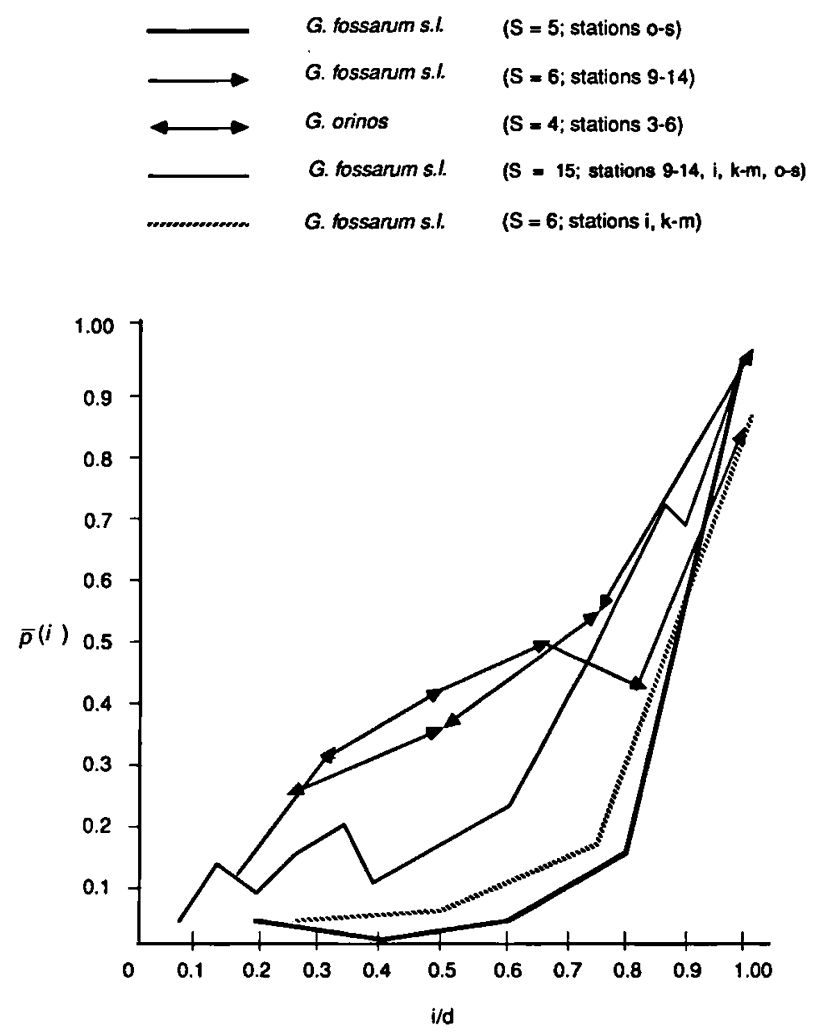

Fig. 5. CAF gene flow profiles (Slatkin, 1981) in subsets of $G$. fossarum s.l. and G. orinos. For legend see Fig.4.

of $8.08,1.17$ and 1.75 respectively; Table XIII shows that pairwise combinations of individual populations may yield high $N m_{\theta}$-values up to 11.38. Geographic distances in these combinations range from 4 to $65 \mathrm{~km}$ and in combinations for which $N m<1$ from 3 to $55 \mathrm{~km}$. These data indicate that even if the possibility of gene flow seems to exist between individual populations and areas, substantial barriers to genetic exchange may be present over small geographic ranges.

CAF gene flow profiles (Slatkin, 1981; see also Caccone, 1985; Caccone \& Sbordoni, 1987; Waples, 1987) of G. p. pulex, G. orinos, G. cf. fossarum, G. wautieri and $G$. fossarum s.l. are shown in Figs. 4, 5. The diagram of Fig. 6 represents typical patterns of gene flow (Slatkin, 1981; Waples, 1987). With reference to this diagram, in Fig. 4, low gene flow levels occur in $G$. orinos, G. cf. fossarum, G. fossarum s.l., and G. wautieri; and a medium gene flow level in G. p. pulex. All species but 


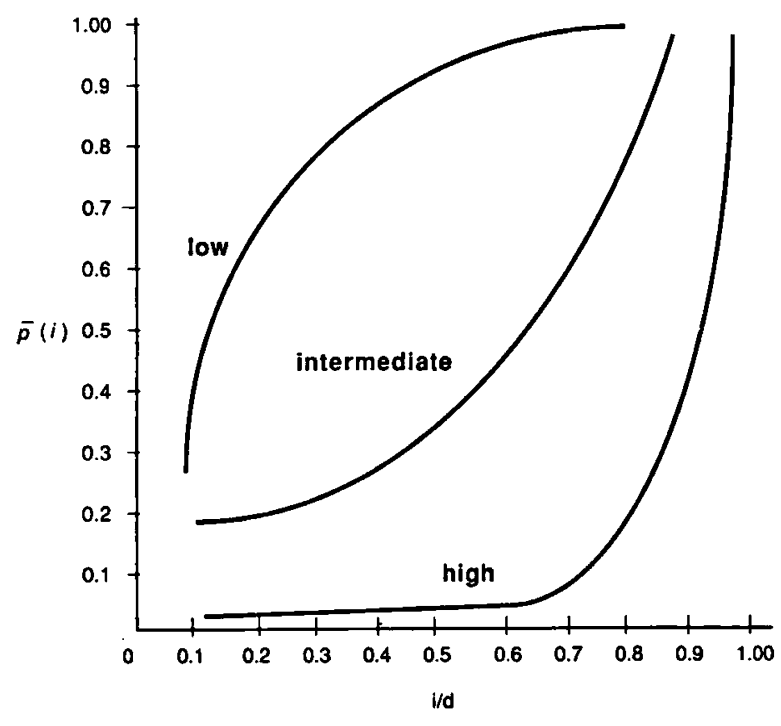

Fig. 6. Typical CAF gene flow profiles in species with the indicated levels of gene flow (after Slatkin, 1981; redrawn from Waples, 1987).

G. p. pulex are characterized by high initial $\bar{p}(i)-$ values, and sometimes by wide fluctuations, typical for low gene flow species (Caccone \& Sbordoni, 1987). Subsampling (Fig. 5) results in a somewhat different pattern. A low to intermediate gene flow level is found in G. fossarum s.l. from areas B and C (stations 9-14); intermediate gene flow levels occur in the combined $G$. fossarum s.l. subset from areas B, C, J, and L (stations 9-14, i, k-m, o-s) and $G$. orinos, whereas gene flow levels in $G$. fossarum s.l. from area $\mathrm{J}$ (stations $\mathrm{o}-\mathrm{s}$ ) and $G$. fossarum s.l. from areas $\mathrm{K}$ and $\mathrm{L}$ (stations $\mathrm{i}, \mathrm{k}-\mathrm{m}$ ) are high. In these curves, fluctuations are less pronounced.

\section{Cross-breeding experiments}

The results of the cross-breeding experiments are listed in Table XIV. All crosses among populations of $G$. fossarum s.l. proved to be completely interfertile. All crosses involving females of $G$. fossarum s.l. from stations $\mathrm{r}, \mathrm{l}$ and $\mathrm{k}$ and males of $G$. cf. fossarum from station $\mathrm{b}$ gave $\mathrm{F} 1$ of fspring; all these crosses but one $\left(f_{k} \times \mathrm{cf} . f_{b}\right.$; females of $G$. fossarum s.l. from station k and males $G$. cf. fossarum from station $b$ ) failed to produce $F 2$ offspring. In reciprocal crosses, one cross (cf. $f_{b} \times f_{r}$; females $G$. cf. fossarum from station b and males of $G$. fossarum s.l. from station $\mathrm{r}$ ) gave $\mathrm{Fl}$ offspring (one female), but no F2 was obtained. As for crosses between populations of $G$. orinos and $G$. fossarum s.l. (including reciprocal crosses), two crosses ( $o_{a}$ $\times f_{l}$, females $G$. orinos from station a and males G. fossarum s.l. from station 1 ; and $f_{k} \times o_{a}$, females $G$. fossarum from station k and males $G$. orinos from station a) gave $\mathrm{F} 1$ offspring in very low numbers; only once $\left(f_{k} \times o_{a},\right), \mathrm{F} 2$ was obtained. In crosses between populations of $G$. orinos and $G$. cf. fossarum, one female from cross cf. $f_{b} \times o_{a}$ gave F1 offspring, but no F2 was obtained.

\section{Discussion and conclusions}

\section{Genetic differentiation}

\section{G. p. pulex \& G. p. gallicus}

Inferring genetic relationships from protein polymorphisms in the $G$. pulex-group resulted in some unexpected patterns. The topology of genetic structures was generally determined by geographic distribution, but sometimes highly incongruent with patterns of morphology. Morphologically defined taxa covering a large geographic area may show little differentiation, while others are highly structured over much shorter distances.

Considering the various levels of genetic differentiation among the populations and species studied in both UPGMA dendrogram and Wagner tree (Figs. 2, 3), the low levels of divergence among populations of G. p. pulex and G. p. gallicus are striking. Intra-specific $I$-values of $G$. pulex (i.e. $G$. p. pulex + G. p. gallicus) range from $0.89-0.99$ $\left(I_{\text {mean }}= \pm 0.94 ; D_{\min }-D_{\max }=0.01-0.11 ; D_{\text {mean }}\right.$ $= \pm 0.06$ ). With reference to other taxa (e.g. Scheepmaker et al., 1988; Scheepmaker \& Van Dalfsen, 1989), I-values of comparisons of geographically distant populations of G.p.pulex and G. p. gallicus may be remarkably high. For instance, G. p. gallicus from station 23 in the Pyrenees (southern France; Table I, Fig. 1A, G), and $G$. p. pulex from station t (the Netherlands; Table I, Fig. 1A) yield an $I$-value of $0.93(D=$ 0.07). G. p. pulex has an Eurasiatic distribution 
(Pinkster, 1972; Karaman \& Pinkster, 1977); in western Europe it is found from southern Scandinavia to southern France. According to Thienemann (1950), the extent of the distribution area makes a postglacial dispersion highly improbable. Therefore, G. p. pulex is to be considered as a preglacial, original inhabitant of the lower reaches of rivers and streams in the central European lowlands as defined by Thienemann (1950). The level of divergence between G. p. pulex and G. p. gallicus is likely to be of preglacial origin (Figs. 2 and 3). During the Pleistocene glaciations G. p. gallicus (at present restricted to southern France; see Table I) probably subsisted in the Mediterranean refugial zone, whereas $G$. $p$. pulex re-invaded western European lowlands in postglacial times.

\section{G. wautieri}

Roux (1971) demonstrated that $G$. wautieri was reproductively isolated from $G$. p. pulex and $G$. fossarum s.l. Due to an important overlap of discriminating morphological characters, he had difficulties to distinguish the younger individuals (especially females) of $G$. wautieri from $G$. p. pulex and $G$. fossarum s.l. Attempts to support the specific status of $G$. wautieri by other means, such as caryological and chromatographical techniques, were unsuccessful. Based upon protein polymorphisms however, $G$. wautieri forms a well-defined cluster, clearly distinct from G. p. pulex and $G$. fossarum s.l. (Figs. 2, 3). In the present study, only $G$. wautieri from the French Alps was involved. As $G$. wautieri is also known from other areas (e.g. the Massif Central in central France, and N.E. of the Alps) the data presented here are incomplete and may depict a biased intraspecific genetic variation pattern.

\section{G. fossarum s.l.}

Morphologically very similar (and generally con-' sidered conspecific) forms of G. fossarum s.l., G. orinos and $G$. cf. fossarum are occasionally grouped separately in distinct clusters (UPGMA and Wagner dendrogram; Figs. 2, 3). This is in contrast with G. p. pulex, G. p. gallicus, and G. wautieri. Subdivision of morphologically very similar forms belonging to $G$. fossarum s.l. was discussed by
Scheepmaker \& Van Dalfsen (1989). The material studied by these authors (Fig. 1, areas J, K, L; Table I, stations $\mathrm{i}, \mathrm{k}-\mathrm{m}, \mathrm{o}-\mathrm{s}$ ) has been completed by additional sampling in areas B, C, F and $\mathrm{I}$. In the combined data set $G$. fossarum s.l. from areas $\mathrm{J}, \mathrm{K}$, L (except for station j), B/C, I, and stations 17 and o (Table I; Fig. 1A) forms a highly differentiated, but monophyletic group (Figs. 2, 3).

Because of its present day distribution, G. fossarum s.l. is considered a preglacial inhabitant of the Alps (a "southern glacier border form" according to Thienemann, 1950). It is likely that it reached the periphery of its distribution as recorded in this study (e.g. areas $\mathrm{J}$ and $\mathrm{K}$ in Fig. 1A) only since the glacial ages. This theory is supported by the high levels of genetic similarity among $G$. fossarum s.l. populations from areas J, C, L and K (Fig. 1A, Figs. 2, 3).

\section{G. orinos \& G. cf. fossarum}

$G$. orinos and $G$. cf. fossarum are morphologically closely related forms (Scheepmaker et al., 1988). The subdivision of both $G$. orinos (areas D, E, station 3-6, vs. area F, stations a, 1-2) and G. cf. fossarum (area $\mathrm{H}$, stations b-d, vs. area $\mathrm{G}$, stations 7 , 8) coincides with an Atlantic-Mediterranean watershed (Figs. 2, 3). This subdivision results in 4 genetically more or less equidistant population sets. Levels of genetic differentiation among these subsets $(I=0.63-0.87 ; D=0.14-0.39)$ are about the same as between each of these subsets and subsets of $G$. fossarum s.l. or G. stupendus ( $I=$ $0.73-0.83$ and $0.74-0.84$, respectively). Crossbreeding experiments show that $G$. cf. fossarum from area $\mathrm{H}$ (station b) and $G$. orinos from area $\mathrm{F}$ (station a) are reproductively isolated (Table XIV; $I=0.67-0.87)$. Unfortunately, $G$. orinos from areas $\mathrm{D} / \mathrm{E}$ and $G$. cf. fossarum from area $\mathrm{G}$ were not involved in these crosses. However, crosses of G. orinos (station a, area F) and G. cf. fossarum (area $\mathrm{H}$, station b) with $G$. stupendus $(I=0.82$ and 0.75 , respectively) and $G$. fossarum s.l. (stations k, $\mathrm{l}, \mathrm{r} ; I=0.81,0.80,0.84$ and $0.69,0.70,0.70$, respectively) were not very successful (Table XIV). Differences between these taxa and $G$. orinos from areas $\mathrm{D} / \mathrm{E}$, and $G$. cf. fossarum from area $\mathrm{G}$ are in the same order of magnitude. $G$. orinos from areas $\mathrm{D} / \mathrm{E}$ and $\mathrm{F}$ (yielding the highest $\boldsymbol{I}$-values recorded 
Table XIV. Results of cross-breeding experiments between samples of G. fossarum s.l., G. orinos and G. cf. fossarum. $f_{r}=G$. fossarum s.l. from station $\mathrm{r} ; f_{l}=$ idem, from station l; $f_{k}=$ idem, from station k; $o_{a}=G$. orinos from station a; cf. $f_{b}=G$. cf. fossarum from station b; legend stations cf. Table I. ${ }^{*}$ ) = crosses by Pinkster \& Scholl (1984).

\begin{tabular}{|c|c|c|c|c|c|c|}
\hline$\rho^{C}$ & $\begin{array}{l}\text { Cross } \\
\times \circ \sigma\end{array}$ & Number of $९$ & Number of $\sigma^{\circ} \sigma^{\circ}$ & $\begin{array}{l}\text { Number of } \\
\text { ovigerous } ९ \text { ? }\end{array}$ & $\begin{array}{l}\text { Number of ovig. } \\
९ \uparrow \text { with offspring }\end{array}$ & $\begin{array}{l}\text { F2 } \\
\text { obtained }\end{array}$ \\
\hline$f_{r}$ & $\times f_{r}$ & 32 & 32 & 15 & 10 & + \\
\hline$f_{l}$ & $\times f_{l}$ & 126 & 122 & 81 & 18 & + \\
\hline$f_{k}$ & $\times f_{k}$ & 102 & 107 & 51 & 17 & + \\
\hline$o_{a}$ & $\times o_{a}$ & 107 & 77 & 60 & 12 & + \\
\hline cf. $f_{b}$ & $\times \mathrm{cf} . f_{b}$ & 167 & 114 & 93 & 20 & + \\
\hline$f_{l}$ & $\times f_{r}$ & 200 & 135 & 67 & 23 & + \\
\hline$f_{l}$ & $\times f_{k}$ & 114 & 115 & 65 & 26 & + \\
\hline$f_{l}$ & $\times o_{a}$ & 57 & 47 & 38 & - & - \\
\hline$f_{l}$ & $\times \mathrm{cf} . f_{b}$ & 163 & 112 & 89 & 1 & - \\
\hline$f_{k}$ & $\times f_{r}$ & 192 & 119 & 117 & 27 & + \\
\hline$f_{k}$ & $\times \mathrm{cf} . f_{b}$ & 114 & 89 & 69 & 11 & + \\
\hline$\hat{f_{k}}$ & $\times o_{a}$ & 122 & 99 & 83 & 2 & + \\
\hline$f_{k}$ & $\times f_{l}$ & 61 & 61 & 51 & 18 & + \\
\hline$o_{a}$ & $\left.\times f_{r}^{*}\right)$ & & & & & \\
\hline$o_{a}$ & $\times f_{l}$ & 82 & 52 & 12 & 1 & - \\
\hline$o_{a}$ & $\times f_{k}$ & 104 & 104 & 45 & - & - \\
\hline$o_{a}$ & $\times$ cf. $f_{b}$ & 197 & 214 & 131 & 1 & - \\
\hline cf. $f_{b}$ & $\times f_{r}$ & 230 & 190 & 34 & 1 & - \\
\hline cf. $f_{b}$ & $\times f_{l}$ & 210 & 182 & 83 & - & - \\
\hline cf. $f_{b}$ & $\times f_{k}$ & 155 & 126 & 99 & - & - \\
\hline cf. $f_{b}$ & $\times o_{a}$ & 199 & 144 & 72 & - & - \\
\hline$f_{r}$ & $\times f_{l}$ & 142 & 87 & 110 & 37 & + \\
\hline$f_{r}$ & $\times f_{k}$ & 248 & 100 & 182 & 46 & + \\
\hline$f_{r}$ & $\left.\times o_{a}^{*}\right)$ & & & & & \\
\hline$f_{r}$ & $\times \mathrm{cf} . f_{b}$ & 368 & 207 & 249 & 12 & - \\
\hline
\end{tabular}

among subsets of $G$. orinos) and $G$. cf. fossarum ( $I$ $=0.67-0.87$, possibly evolve as independent units, but they are probably still to be considered conspecific. On the other hand, $\boldsymbol{G}$. cf. fossarum from areas $\mathrm{G}$ and $\mathrm{H}(I=0.63-0.69)$ are likely to represent distinct taxa, apart from $G$. orinos, $G$. fossarum s.l. and G. stupendus. However, subsets of $G$. gauthieri from the Iberian peninsula yielding $I$-values as low as $0.56-0.65$ were shown to be at least partially interfertile (and thus possibly conspecific; Scheepmaker et al., 1988).

\section{G. stupendus}

Subdivision in $G$. stupendus was discussed in detail by Scheepmaker (1987). The three population samples analyzed here (stations 19, 20 and e, belonging to $G$. stupendus forma A, B and C respectively, according to Pinkster, 1983) represent morphologically different and genetically rather discontinuous forms (Scheepmaker, 1987). Morphologically, $G$. stupendus forma $\mathrm{A}$ is very similar to $G$. fossarum s.l.; G. stupendus forma B resembles $G$. cf. fossarum, and $G$. stupendus forma $\mathrm{C}$ resembles $G$. orinos in many respects (Table III).

6. G. fossarum s.str., G. orinos, G. cf. fossarum, G. stupendus, G. wautieri vs. G. p. pulex \& G. fossarum s.l.

G. p. pulex and G. fossarum s.l. are both considered to be preglacial inhabitants of western Europe. Consequently, their level of divergence (Fig. 2) must be of preglacial origin. As G. fossarum s.l. is considered as a preglacial inhabitant of the Alps, inter-areal differentiation (e.g. areas B and $C$ vs. area J; Fig. 2) must have occurred since the Pleistocene glaciations. From these data we may conclude, that species and forms as $G$. fossarum s.l., G. fossarum s.str., G. orinos, G. cf. fossarum, G. stupendus and $G$. wautieri, having an "earlier" level of divergence, are all likely to be of 
preglacial origin. These levels do not necessarily correspond to those in the Wagner network of Fig. 3 , which is only arbitrarily rooted by the midpoint rooting procedure (Farris, 1972); in fact, as will be discussed in the next section, there is evidence for this network to be rooted at a different internodium.

Unlike G. fossarum s.l., which is widely distributed in central and northern France, but confined to the area covered with tundras (Brinkman, 1959) during the last glacial period, $G$. orinos, $G$. cf. fossarum and $G$. stupendus have limited distribution areas in the old Hercynian massifs in southern France. According to Figs. 2 and 3, smaller geographical units (e.g. G. orinos from areas $\mathrm{D}$ and $\mathrm{E}, \boldsymbol{G}$. cf. fossarum from area $\mathrm{G}$ and $\mathrm{H}$ ) partition a greater portion of the total genetic variation than the larger ones (e.g. G. p. pulex, G. fossarum s.l. from areas $\mathrm{B}, \mathrm{C}, \mathrm{J}, \mathrm{K}$ and $\mathrm{L}$ ). The smaller units correspond to the former Mediterranean refugia during the Pleistocene glaciations. In contrast to the tundra belt, environmental "lowland" conditions may still have prevailed in lower reaches of rivers and tributaries. G. orinos, $G$. cf. fossarum and $G$. stupendus generally inhabit the upper reaches of rivers. As upper reaches are generally separated from each other by lower reaches, this may have prevented them from a wider dispersal.

\section{Phylogenetic relationships among the species and forms studied}

The topologies of the dendrograms obtained by the UPGMA method and the distance Wagner procedure (Figs. 2 and 3) are not congruent. They contrast primarily by suggesting different relationships among species and forms; however, the main clusters they distinguish agree quite well.

The UPGMA method and Nei's (1972) distance coefficient require both the assumption of homogeneous evolutionary rates. Moreover, Nei's distance is unsuitable for deriving phylogenies because of its non-metricity (e.g. Farris, 1981). As the distance Wagner method (Farris, 1972) does not make any assumption about evolutionary rates, this method seems more appropriate to infer phylogenetic relationships. However, the midpoint root- ing (Farris, 1972) as applied in Fig. 3, also assumes homogeneous evolutionary rates. The complete data set of the western European members of the $G$. pulex-group (i.e. the data from this study linked to data sets from the Iberian peninsula and Morrocco; Scheepmaker et al., 1988; Scheepmaker, 1990) in fact suggests that the Wagner network should not be rooted at the midpoint, but at the internodium between G. fossarum s.str. and G. fossarum s.l. from station $\mathrm{j}$ (Fig. 3). In a forthcoming paper, the phylogeny of western European members of the $G$. pulex-group, derived from protein polymorphisms, will be discussed in more detail.

Comparison of methods used for analyzing population structure and estimating levels of gene flow

The methods used to assess levels of gene flow differ in the assumption they depend on. The use of $F$-statistics assumes that genetic divergence by stochastic processes is neutralized by the effects of gene flow, and not by other processes, such as natural selection. Slatkin's methods $(1981,1985)$ do not depend on the assumption of selective neutrality (Waples, 1987). Unlike $F_{S T}$-values according to Wright $(1931,1951,1965)$, the $\theta$-values of Weir \& Cockerham (1984) do not make assumptions concerning numbers of populations, sample sizes or heterozygote frequencies. Therefore, according to these authors, they are suited to small data sets. $N m_{F_{S T}}$ and $N m_{\theta^{-}}$-values are closely correlated, yielding a correlation coefficient $(r)$ of 0.99 , $N m_{F_{S T}}$ overestimates levels of gene flow with regard to $\mathrm{Nm}_{\theta}$, possibly due to incorrect assumptions about sample sizes, etc. (however, in simulations of Slatkin \& Barton, 1989, both methods performed equally well for moderate levels of gene flow).

In all but one cases there is a rather close agreement between $N m^{*}$ - and $N m_{\theta}$-values. When the deviating $\mathrm{Nm}^{*}$-value is omitted, $\mathrm{Nm}^{*}$ and $N m_{\theta}$ yield a correlation coefficient of 0.97 ; for estimates that were not corrected for sample size, this value is 0.93 . The deviating high $N m^{*}$-value ( $G$. fossarum s.l.; Table XII) can be attributed to the greater number of samples involved (about three times more than in the other subsets). In fact, the in- 
fluence of high frequencies of $\bar{p}(1)$ in areas B and $C$ are neutralized by averaging them with the much lower $\bar{p}$ (1)-frequencies in areas $\mathrm{J}, \mathrm{K}$ and $\mathrm{L}$; moreover, as the number of populations increases, a number of alleles that were private may not be private anymore. One might argue that the complete data set of $G$. fossarum s.l. (19 populations; $G$. fossarum s. str is not included) yields a very low $\mathrm{Nm}^{*}$-value; however, this value is strongly affected by some $\bar{p}(1)$-values of 1 , due to genetic isolates. It may be concluded that we should only compare $\mathrm{Nm}^{*}$ estimates of data sets of about the same number of populations.

As stressed by Caccone \& Sbordoni (1987), Slatkin's (1985) method is appropriate for identifying cases in which the majority of populations sampled are exchanging (or recently exchanged) genes at a rather high rate, but a few populations are genetic isolates.

In many sets and subsets of population samples, the $\theta$-value of many polymorphic loci is significantly greater than 0 (Tables VIII-X). It is unlikely, that all those loci are affected by natural selection at the same time. The correlation between $N m_{\theta}$ and $\mathrm{Nm}^{*}$ (presumed not to be influenced by natural selection; Barton \& Slatkin, 1987) may be in favour of this view. As pointed out by Slatkin (1985), a basic problem (which, although for different reasons, also affects $\mathrm{Nm}$ estimates obtained with $\mathrm{F}$ statistics) is the effect on the estimated value of $\mathrm{Nm}^{*}$ when migration is between nearest neighbours in a stepping stone model, and the populations sampled are separated by very large distances (e.g. $G$. p. pulex). In that case, an allele that is encountered in only one population could be present in several adjacent populations that were not sampled. A minimum number of samples, relative to the area covered and its structure and to the presumed dispersal abilities of the species under study, is recommended. Spatial heterogeneity may also be a source of bias in estimating levels of gene flow. Splitting up the dataset of a species in different subsets also contributes to a more accurate $\mathrm{Nm}$ estimate. Another problem is the occurrence of bottlenecks. When gene flow levels are low $(\mathrm{Nm}<1)$, rare alleles will disappear due to genetic drift and bottlenecks. Consequently, $\mathrm{Nm}^{*}$ will overestimate the ac- tual levels of gene flow, representing rather historical patterns of genetic exchange.

\section{Population structures of morphological species}

G. p. pulex and G. p. gallicus show little genetic differentiation, although the geographical area covered by these forms is large. The gene flow profile of G. p. pulex (Fig. 4) resembles the characteristic curve of high gene flow species. However, G. $p$. pulex is significantly subdivided at most loci (Table IX) and estimated gene flow levels $\left(\mathrm{Nm}^{*}\right.$ and $\left.N m_{\theta}\right)$ are low (less than one; Table XI). The reasons may be twofold. Firstly (probably the most important), due to the small number of samples (and small sample sizes) relative to the large area covered, a basically clinal variation pattern will appear discontinuous, and consequently, estimated levels of gene flow will be low. Secondly, low levels of genetic differentiation and low gene flow profiles (CAF) may be more indicative for historical patterns of genetic exchange than of current patterns, whereas the present distribution of allelic variants indicates that genetic exchange in the past may have been higher.

Contrary to $G$. p. pulex, populations of $G$. fossarum s.l., G. orinos, G. cf. fossarum and $G$. stupendus in fact are significantly subdivided at many polymorphic loci. Significant subdivision even occurred at higher levels than those recorded in cave-dwelling species (Caccone \& Sbordoni, 1987), and estimated levels of gene flow $\left(\mathrm{Nm}^{*}\right.$ and $N m_{\theta}$ ) are generally smaller than one. An explanation is possibly provided by the (in)stability of the environment. Although isolated, the cave environment (and consequently population sizes) may be stable over many years. On the other hand, streams and rivers (especially in the Alps and the Mediterranean area) may be subject to dramatic environmental changes, such as almost complete desiccation (Scheepmaker, 1987) or strongly increased discharge and subsequent erosion due to heavy rainfall. Repeatedly, surviving population fragments are subjected to stochastic processes. This hypothesis is supported by area dependent gene flow levels in G. fossarum s.l. (Table XII). Levels of gene flow 
in the French Alps (areas B, C) are low; in northern France however (area J), where moderate environmental conditions prevail, levels of gene flow are higher $\left(\mathrm{Nm} *\right.$ and $N m_{\theta}$ are substantially $\left.>1\right)$. As a matter of fact, Goedmakers (1981) and Goedmakers \& Pinkster (1981), who demonstrated that gammarids are actively and sometimes massively migrating organisms, studied G. fossarum s.l. in this very area. However, this activity may be quite different from one drainage system to another (Pinkster, pers. comm.). The observed population subdivision in structured environments agrees with findings of Gooch \& Hetrick (1979) and Scheepmaker (1987), who studied freshwater gammarids in karst areas; these authors also reported sharp discontinuities in population structure.

\section{Estimated levels of gene flow and environmental structure}

The results reported here confirm that gene flow occurs primarily in relation to environmental structure. For example, in the highly structured French Alps (areas B, C) the $\mathrm{Nm}^{*}$ estimate within $\mathrm{G}$. fossarum s.l. is 0.20 ; in area $\mathrm{J}$, a more uniform area, this value is 1.51 .

For comparison, within the genus Gammarus, between two population samples of the freshwater form $G$. duebeni celticus in Brittany (France) the $N m_{\theta}$ estimate is 0.60 ; between two population samples of the euryhaline brackish form $G$. $d$. duebeni from area J (Fig. 1A) and Petten (North-Holland) this estimate yields 5.98 (Scheepmaker, 1990); in a marine environment, $\mathrm{Nm}_{\theta}$ estimates of $\boldsymbol{G}$. salinus (18 samples, ranging from Denmark to southern Brittany) and subsets of G. zaddachi (7 and 11 samples, ranging from Tromsø to Brittany) calculated from data provided by Bulnheim \& Scholl (1981) yield 4.26, 7.79 and 1.45, respectively; in a lowland and "polder" environment, G. tigrinus (10 samples from Holland to Denmark; calculated from Bulnheim, 1985) yields an $N m_{\theta}$ estimate of 1.56 (Scheepmaker, unpublished data). These data corroborate the suggested relation between environmental structure and estimated levels of gene flow.
Genetic differentiation and cross-breeding experiments

Cross-breeding experiments by Roux (1971), Goedmakers (1972), Pinkster (1972, 1983), Pinkster \& Scholl (1984), Scheepmaker (1987), and results from crosses presented in this study (Table XIV) suggest that clusters formed by $G$. pulex, G. wautieri, G. stupendus, G. fossarum s.l. from areas J, K, L, B, C, F and I (Fig. 1A), G. orinos from area $\mathrm{F}$, and $G$. cf. fossarum from area $\mathrm{H}$, act as independently evolving evolutionary units. Forms within those clusters are thus to be considered conspecific. The position of $G$. fossarum s.l. from stations $\mathrm{n}$ and j, G. fossarum s. str., G. orinos from areas D and $\mathrm{E}$, and $G$. cf. fossarum from area $\mathrm{G}$ remains uncertain. Although not morphologically distinct from other forms, it seems quite well possible that these forms also evolve independently, ultimately resulting in distinct species.

\section{Acknowledgements}

I wish to thank Dr. Ben Crabtree, Prof. Dr. Steph Menken, Dr. Sjouk Pinkster and Prof. Dr. Jan Stock for commenting on the manuscript, and Prof. Menken for substantial advice concerning $F$ statistic analysis and indirectly estimated levels of gene flow. Special thanks are due to Dr. Willem Ellis for writing the Thèta-program and to Dr. L. Botosaneanu for valuable discussions and correcting the French abstract. I am furthermore indebted to Matthijs van Couwelaar and Marian Goris for their assistance in the field, and to Bart van den Hoek for his help in running the cross-breeding experiments.

\section{References}

Ayala, F.J. \& J.R. Powell, 1972. Allozymes as diagnostic characters of sibling species of Drosophila. Proc. natn. Acad. Sci. USA, 69(5): 1094-1096.

Barton, N.H. \& M. Slatkin, 1987. A quasi equilibrium theory of the distribution of rare alleles in a subdivided population. Heredity, 56: 409-415.

Brinkmann, R., 1959. Abriss der Geologie, 2: 1-360 (Ferdinand Enke Verlag, Stuttgart). 
Bulnheim, H.P., 1985. Genetic differentiation between natural populations of Gammarus tigrinus (Crustacea, Amphipoda) with reference to its range extension in European continental waters. Arch. Hydrobiol., 102: 273-290.

Bulnheim, H.P. \& A. Scholl, 1981. Genetic variation between geographic populations of the amphipods Gammarus zaddachi and G. salinus. Mar. Biol., 64: 105-115.

Caccone, A., 1985. Gene flow in cave arthropods: a qualitative and quantitative approach. Evolution, 39: 1223-1235.

Caccone, A. \& V. Sbordoni, 1987. Molecular evolutionary divergence of North American cave crickets. I. Allozyme variation. Evolution, 41 (6): 1198-1214.

Ellis, W.N., 1989. Thèta version 1.01; a program for the Macintosh computer for the analysis of population structure and the estimation of gene flow levels based on the method of Weir \& Cockerham (1984). Available free of charge from the author.

Farris, J.S., 1972. Estimating phylogenetic trees from distance matrices. Am. Natural., 106: 645-668.

Farris, J.S., 1981. Distance data in phylogenetic analysis. In: V.A. \& D.R. Brooks, eds. Advances in cladistics: Proceedings of the first meeting of the Willi Hennig Society, 3-23 (New York Botanical Garden, Bronx).

Goedmakers, A., 1972. Gammarus fossarum Koch, 1835: Redescription based on neotype material and notes on its local variation (Crustacea, Amphipoda). Bijdr. Dierk., 42 (2): 124-138.

Goedmakers, A., 1974. Les Gammaridae (Crustacés-Amphipodes) du Massif Central. Bull. zool. Mus. Univ. Amsterdam, 3 (23): 211-220.

Goedmakers, A., 1981. Population dynamics of three gammarid species (Crustacea, Amphipoda) in a French chalk stream, 4. Review and implications. Bijdr. Dierk., 51 (2): 181-190.

Goedmakers, A. \& S. Pinkster, 1981. Population dynamics of three gammarid species in a French chalk stream, 3. Migration. Bijdr. Dierk., 51 (2): 145-180.

Goedmakers, A. \& A.L. Roux, 1975. Essais d'hybridation entre plusieurs populations de Gammares du groupe pulex (Amphipoda). Crustaceana, 24 (1): 99-109.

Gooch, J.L. \& S.W. Hetrick, 1979. The relation of genetic structure to environmental structure: Gammarus minus in a karst area. Evolution, 33: 192-206.

Karaman, G.S. \& S. Pinkster, 1977. Freshwater Gammarus species from Europe, North Africa and adjacent regions of Asia (Crustacea, Amphipoda). Part 1. Gammarus pulex group and related species. Bijdr. Dierk., 47 (1): 1-97.

Nei, M., 1972. Genetic distance between populations. Am. Natural., 106: 283-292.

Pinkster, S., 1972. On members of the Gammarus pulex-group (Crustacea, Amphipoda) from western Europe. Bijdr. Dierk., 42 (2): 164-191.

Pinkster, S., 1983. The value of morphological characters in the taxonomy of Gammarus. Beaufortia, 33 (2): 15-28.

Pinkster, S. \& A. Scholl, 1984. Gammarus orinos n. sp. from the Massif Central (France): its genetic and morphological distinction from Gammarus ibericus Margalef, 1951 (Crustacea, Amphipoda). Bijdr. Dierk., 54 (1): 139-146.

Rogers, J.S., 1972. Measures of genetic similarity and genetic distance. Studies in Genetics, Univ. Texas Publ., 7213: 145-153.

Roux, A.L., 1971. Les Gammares du groupe pulex. III. Essais d'hybridation et examen caryologique. Archs. Zool. exp. gén., 112: 819-868.

Scheepmaker, M., 1987. Morphological and genetic differentiation of G. stupendus Pinkster, 1983 in the Massif de la Sainte Baume, France. Bijdr. Dierk., 57 (1): 1-18.

Scheepmaker, M., 1990. Genetic differentiation, origin and dispersal of Gammarus gauthieri from the Iberian peninsula and North Africa (Crustacea, Amphipoda). Bijdr. Dierk., 60 (1): 31-49.

Scheepmaker, M. \& J. van Dalfsen, 1989. Genetic differentiation in Gammarus fossarum and G. caparti (Crustacea, Amphipoda) with reference to G. pulex pulex in northwestern Europe. Bijdr. Dierk., 59 (3): 127-139.

Scheepmaker, M., F. van der Meer \& S. Pinkster, 1988. Genetic differentiation of the Iberian amphipods Gammarus ibericus Margalef, 1951 and G. gauthieri S. Karaman, 1935, with reference to some related species in France. Bijdr. Dierk., 58 (2): $205-226$.

Slatkin, M., 1981. Estimating levels of gene flow in natural populations. Genetics, 99: 323-335.

Slatkin, M., 1985. Rare alleles as indicators of gene flow. Evolution, 39: 53-65.

Slatkin, M. \& N.H. Barton, 1989. A comparison of three indirect methods for estimating average levels of gene flow. Evolution, 43 (7): 1349-1368.

Sneath, P.H.A. \& R.R. Sokal, 1973. Numerical taxonomy: i-xv, 1-780 (W.H. Freeman and Company, San Francisco).

Swofford, D.L. \& R.B. Selander, 1981. Biosys-1. A computer program for the analysis of allelic variation in genetics. $J$. Heredity, 72: 281-283.

Thienemann, A., 1950. Verbreitungsgeschichte der Süsswassertierwelt Europas. Binnengewässer, Stuttgart, 18: [i-xiv], 1-809.

Waples, R.S., 1987. A multispecies approach to the analysis of gene flow in marine shore fishes. Evolution, 41: 385-399.

Wautier, J. \& A.L. Roux, 1959. Note sur les Gammares du groupe pulex dans la région Lyonnaise. Bull. mens. Soc. linn. Lyon, 28 (3): 76-83.

Weir, B.S. \& C.C. Cockerham, 1984. Estimating F-statistics for the analysis of population structure. Evolution, 38 (6): 1358-1370.

Workman, P.L. \& J.D. Niswander, 1970. Population studies in southwestern Indian tribes. II. Local genetic differentiation in the Papago. Amer. J. human Genet., 22: 24-49.

Wright, S., 1931. Evolution in Mendelian populations. Genetics, 16: 97-159.

Wright, S., 1951. The genetical structure of populations. Ann. hum. Genet., 15: 323-354.

Wright, S., 1965. The interpretation of population structure by F-statistics with special regard to systems of mating. Evolution, 19: 395-420.

Received: 5 May 1989

Revised: 9 January 1990 